DOI: 10.1002/ ((please add manuscript number))

Article type: Full Paper

\title{
Overcoming the Cut-off Charge Transfer Bandgaps at the PbS Quantum Dot Interface
}

Ala'a O. El-Ballouli, Erkki Alarousu, Ahmad R. Kirmani, Aram Amassian, Osman M. Bakr, and Omar F. Mohammed*

A. El-Ballouli, Dr. E. Alarousu, A. Kirmani, Prof. A. Amassain, Prof. O. M. Bakr, Prof. O. F. Mohammed.

Division of Physical Sciences and Engineering

Solar and Photovoltaics Engineering Research Center

King Abdullah University of Science and Technology (KAUST)

Thuwal 23955-6900, Saudi Arabia

E-mail: omar.abdelsaboor@kaust.edu.sa

Keywords: quantum dots, photovoltaic devices, charge carrier injection, time-resolved spectroscopy, interfacial electrostatic interaction

Light harvesting from large size of semiconductor $\mathrm{PbS}$ quantum dots (QDs) with a band gap of less than $1 \mathrm{eV}$ is one of the greatest challenges precluding the development of PbS QDbased solar cells because the interfacial charge transfer (CT) from such QDs to the most commonly used electron acceptor materials is very inefficient, if it occurs at all. Thus, an alternative electron-accepting unit with a new driving force for CT is urgently needed to harvest the light from large-sized PbS QDs. Here, we utilize a cationic porphyrin as a new electron acceptor unit with unique features that bring the donor-acceptor components into close molecular proximity, allowing ultrafast and efficient electron transfer for QDs of all sizes, as inferred from the drastic photoluminescence (PL) quenching and the ultrafast formation of the porphyrin anionic species. Our time-resolved results clearly demonstrate the possibility of modulating the electron transfer process between $\mathrm{PbS}$ QDs and porphyrin moieties not only by the size quantization effect but also by the interfacial electrostatic interaction between the positively charged porphyrin and the negatively charged QDs. This approach provides a new pathway for engineering QD-based solar cells that make the best use of the diverse photons making up the Sun's broad irradiance spectrum. 


\section{WILEY-VCH}

\section{Introduction}

One of the most important scientific and technological challenges of this century is the design of cost-effective next-generation photovoltaics (PVs) that operate efficiently over the broad solar irradiance spectrum range. Quantum dot (QD) solar cells, ${ }^{1}$ especially those based on $\mathrm{PbS}$ QDs, have emerged as promising candidates because of QDs' size-tunable bandgaps $\left(\mathrm{E}_{\mathrm{g}}\right)$, high absorptivity coefficients, solution-processability, and facile synthesis. Moreover, these QDs exhibit a unique ability to increase the photon conversion efficiency (PCE) beyond the theoretical Shockley-Quiesser (S-Q) limit via processes such as multiple exciton generation (MEG) and hot-carrier extraction..$^{2-4}$ These features of PbS QDs have enabled the engineering of various single-junction PV device architectures based on this material ${ }^{5-7}$ in addition, researchers have elucidated the charge transfer $(\mathrm{CT})$ rates between $\mathrm{PbS}$ QDs and various acceptor moieties $^{8-11}$ to optimize their PV performance. Additionally, researchers attempting to fabricate tandem solar cells based on the size-quantization effect of $\mathrm{PbS}$ QDs have thus far successfully utilized QDs with E $_{\mathrm{g}}$ values of 1.6 and $1.0 \mathrm{eV}$ to boost the PCEs. ${ }^{12,13}$ Similarly, because of the additional driving force of quantum funneling, researchers have enhanced the PCE by employing a graded device architecture using PbS QDs with intermediate $\mathrm{E}_{\mathrm{g}}$ values between 1.35 and $1.06 \mathrm{eV} .{ }^{14}$ Although the use of larger QDs (with smaller $E_{\mathrm{g}}$ values) remains necessary and a key component to improve the device performance including Tandem solar cells, most of the studies reported to date have demonstrated that only small PbS QDs (core diameter $\leq 4.3 \mathrm{~nm}, \mathrm{E}_{\mathrm{g}} \geq 1.0 \mathrm{eV}$ ) exhibit effective $\mathrm{CT}$ to common electron scavengers such as $\mathrm{TiO}_{2}$ nanoparticles, ${ }^{15} \mathrm{SnO}_{2}$ nanoparticles $,{ }^{16} \mathrm{PCBM},{ }^{11,17}$ and methyl viologen, ${ }^{18}$ which is explained by such QDs exhibiting type II interfacial band alignments. These findings are further supported by a considerable reduction in the device PCE when QDs with larger

diameters are used; ${ }^{19,20}$ thus limiting the energy harvested from the Sun's broad spectrum. 
In this work, we report a key variable component—specifically, electrostatic interaction—as a new driving force for CT at QD interfaces. Porphyrin-based electron acceptors were chosen for this study because of their wide use in PVs, their suitable reduction potentials, ${ }^{21}$ and their peripheral substituents' versatility. The presence or absence of electrostatic interactions between the negatively charged QD surface and the positively or neutrally charged porphyrin derivatives enable the CT to be tuned from highly efficient and ultrafast ( $<120$ fs) to nearly absent (Scheme 1). We demonstrate this tuning effect by manipulating these key parameters. As a result, we have extended the effective QD size for CT to include most of the nearinfrared spectral region, reaching beyond the previously reported cut-off $\mathrm{CT}$ bandgaps for $\mathrm{PbS}$ QDs.

\section{Results and Discussion}

\subsection{Negatively-Charged PbS QDs in Polar Medium}

For this study, halide-passivated PbS QDs were prepared according to the method of Ip et al., ${ }^{5}$ followed by the ligand exchange of oleic acid ligands for 1-thioglycerol (TG) and dispersion in dimethyl sulfoxide (DMSO), as previously reported. ${ }^{22}$ Further synthetic details are available in the Supporting Information (SI). Notably, the halide treatment step $\left(\text { via } \mathrm{CdCl}_{2}\right)^{5,23}$ was critical for better passivation of the surface, thus preserving the QDs' carrier lifetime after ligand exchange (Figure 1A). Without $\mathrm{CdCl}_{2}$-treatment, the QDs showed lower photoluminescence and an increased recovery (recombination of charge carriers) due to trap states and imperfectly passivated surface upon ligand exchange. The ligand exchanged $\mathrm{CdCl}_{2}$ -treated QDs were characterized by X-ray photoelectron spectroscopy (XPS) which confirmed the binding of $\mathrm{Cd}, \mathrm{Cl}$ and TG to the PbS QDs (Figure 1C and 1D, Table S1), in good agreement with previous reports. ${ }^{5,22}$ Transmission electron microscopy (TEM) and dynamic light scattering (DLS) measurements showed the maintenance of a uniform spherical shape and size distribution (Figure S2, Table S2). Further, Zeta-potential measurement 


\section{WILEY-VCH}

(Figure 1B) revealed a negatively-charged surface for these QDs, in agreement with previous report by Sargent and co-workers. ${ }^{22}$ It is worth pointing out that a possible explanation for the more negative values obtained with our QDs could be the extra passivation step, in which $\mathrm{Cl}^{-}$ ions were proven to constitute a higher percentage than $\mathrm{Cd}^{+2}$ ions, as indicated clearly in our XPS measurements (Table S1). We synthesized QDs with different first excitonic absorption peaks $(840,1000,1300,1550 \mathrm{~nm})$ corresponding to $\mathrm{E}_{\mathrm{g}}$ between $1.48 \mathrm{eV}-0.80 \mathrm{eV}$. Herein, we refer to the QD samples based on the wavelength of the $1 S_{h} \rightarrow 1 S_{e}$ transition; e.g., the sample with a $1 \mathrm{~S}_{\mathrm{h}} \rightarrow 1 \mathrm{~S}_{\mathrm{e}}$ peak at $840 \mathrm{~nm}$ is named $\mathrm{PbS}-840$.

\subsection{PbS QDs/Cationic Porphyrin Interface}

Figure 2 shows the changes in the steady-state absorption and photoluminescence (PL) spectra of $\mathrm{PbS}-840$ and $\mathrm{PbS}-1300$ upon the addition of different concentrations of the positively charged free-base porphyrin 5,10,15,20-tetra( $N$-methyl-4-pyridyl)porphyrin (TMPyP), whose structure and absorption spectrum are depicted in the SI (Figure S3A). The successive addition of TMPyP resulted in efficient quenching of the PL of the QDs. This result suggests that photoinduced CT occurs between the QDs as the donor and TMPyP as the acceptor. It is worth pointing out that although fluorescence quenching could also be achieved by energy transfer, we rule out this mechanism here on the basis of the lack of spectral overlap between the absorption of TMPyP and the emission of QDs. For PbS-840, the addition of $0.2 \mathrm{mM}$ TMPyP reduced the PL emission to nearly nil and resulted in a slight blue shift of both the first exciton absorption peak (approximately $15 \mathrm{~nm}$ ) and the PL peak (approximately $12 \mathrm{~nm}$ ). These spectral shifts may originate from modifications in the electronic density and confinement energy of the QDs, which are characteristic of the groundstate complex between a ground-state fluorophore and a quencher, ${ }^{24,25}$ in agreement with previous reports. ${ }^{11}$ Unlike our previous study between oleic acid-capped PbS QDs and PCBM, ${ }^{11}$ large TG-capped QDs (PbS-1300) exhibit complete PL quenching with TMPyP (Figure 2). Although the extents of the spectral shifts were negligible compared to those of 


\section{WILEY-VCH}

PbS-840, the significant PL quenching implies efficient electronic coupling between TMPyP and $\mathrm{PbS}-1300$ in the excited state. The minimized spectral shifts upon TMPyP addition to larger QDs could be the result of the lessened electronic coupling in the ground state (Figure S8) because of the decreased free-energy driving force $(-\Delta G)$ for the CT (Figure S3B). It is worth mentioning that the energy levels of the QDs were estimated with the aid of ultraviolet photoelectron spectroscopy (UPS) measurements to take into account the effect of the ligandinduced modifications on the absolute energy levels of the QDs. ${ }^{26}$ Interestingly, drastic PL quenching was also observed upon the addition of TMPyP to PbS-1550 $\left(\mathrm{E}_{\mathrm{g}}=0.8 \mathrm{eV}\right.$, core diameter $\sim 6 \mathrm{~nm}$, Figure S4). Importantly, this is the first time to report CT from such size of $\mathrm{PbS}$ QDs to molecular acceptor. A further consideration for donor-acceptor systems in the solution phase is whether PL quenching occurs via 1) static quenching, whereby a nonluminescent ground-state complex is formed, and/or 2) dynamic quenching, which involves collision. In this regard, the Stern-Volmer (SV) plots of small and large QDs revealed upward curvatures (Figure S5), thus indicating the incorporation of both quenching mechanisms depending on the [TMPyP]. ${ }^{27,28}$

Next, we employed femtosecond transient absorption (fs-TA) spectroscopy to study the ground-state bleach (GSB) dynamics upon bandgap excitation of the QDs. Because the GSB signal is directly associated with the electron population in the conduction band, we used it as a convenient probe to follow the $\mathrm{CT}$ and charge recombination $(\mathrm{CR})$ dynamics at the $\mathrm{QD}$ interface. In particular, in addition to probing the formation of the porphyrin anionic species, we used the GSB signal to follow the charge recombination dynamics resulting from the CT process. The TA measurements of different PbS QD sizes were recorded following laser pulse excitation at approximately 1.3 times the bandgap to avoid any contribution from the scattered light in the GSB signal, thus assuring high-quality data and accurate dynamics for the CT process. Additionally, low pump energies were maintained, with an average number of photons absorbed per QD less than 0.2, to ensure single-photon absorption and prevent 


\section{WILEY-VCH}

contributions from Auger decay pathways. ${ }^{29}$ Further details of the TA experimental apparatus and conditions are provided in the SI. Figure 3A shows the fs-TA spectra of large QDs (PbS1300) recorded as [TMPyP] was increased. The pristine PbS QDs exhibited a very small decay $(<15 \%)$ of the GSB on the picosecond (ps) time scale, which is attributed to state trapping (which lies just below the first confined electron state) ${ }^{30}$ these results are consistent with those of previous reports ${ }^{4,10}$ and indicate that most excited QDs are in long-lived single exciton states under these experimental conditions. The addition of TMPyP to solutions of $\mathrm{PbS}$ QDs accelerates the GSB recovery on the picosecond time scale. The boosted bleaching recoveries suggest the presence of an alternate deactivation pathway for the separated charges: $\mathrm{CR}$ following a CT process. On the basis of the energy-band alignments shown in Figure S3B, assisted by electrostatic interaction between the negatively-charged QDs surface and the positively charged molecular acceptor, we attribute the exciton quenching to the ultrafast electron transfer from the photo-excited $\mathrm{PbS}$ QDs to TMPyP, which is expected to generate TMPyP anion radical species (TMPyP ${ }^{*}$ ) with a characteristic broad peak between 700 and $800 \mathrm{~nm} \cdot{ }^{31,32}$ By monitoring the fs-TA in the visible region for both small and large QDs, we deciphered the signal for electron injection from the photo-excited PbS QDs to TMPyP through the evolution of TMPyP*-, with a temporal resolution of approximately $120 \mathrm{fs}$ (Figure S6). The ultrafast electron injection to TMPyP as inferred from the formation of its anionic species, and the ultrafast CR as inferred from the ultrafast GSB recovery, are further indications of the ground-state complexation and subsequent static CT between the TGcapped PbS QDs and cationic porphyrin. This could also indicate that the molecular acceptor is likely to be adsorbed on the surface of QDs which is consistent with previous reports. ${ }^{4,8,9}$ Figure 4 shows a comparison of the kinetic traces extracted from the GSB feature within the fs-TA spectra of different PbS QD sizes upon the addition of a fixed [TMPyP]. The dynamics were fit to double-exponential functions with time constants of a few to tens of picoseconds, and tens to hundreds of picoseconds. The two observed components suggest the occurrence of 


\section{WILEY-VCH}

two types of donor-acceptor ion pairs with different associated couplings, in accordance with

previous reports on other donor-acceptor systems. ${ }^{11,33,34}$ The fittings for the bleach recoveries of $\mathrm{PbS}-840, \mathrm{PbS}-1000$, and $\mathrm{PbS}-1300$ indicate that not only is the percentage recovery greater for smaller PbS QDs, but the rate of CR is also enhanced with the smaller QD size. For instance, the recovery time constants due to carrier recombination for PbS-840 are 7.4 and $87.5 \mathrm{ps}$ at $0.1 \mathrm{mM}$ TMPyP, and they elongate to 25.8 and $572.2 \mathrm{ps}$ for PbS-1300 with similar [TMPyP], thus indicating that the CR varies strongly as a function of QD size. The differences in the recombination rates/percentages indicate variation in the CT rates between various QD sizes and TMPyP. In addition, the system reveals a clear dependence not only on the free energy driving force between the donor and acceptor units ${ }^{35}$ (Figure S3B); but also on the presence of the charged molecular acceptors. Because of their increased bandgaps, smaller QDs are expected to have more favorable CB energies for injecting electrons into TMPyP. Notably, although we did not measure the GSB recovery kinetics for PbS-1550 because of a lack of sufficient white light in this region within our TA setup, the steady-state PL spectra (Figure S4) provide sufficient evidence for the CT to TMPyP.

To explore the possibility of electron transfer through diffusion control process, we employed nanosecond TA spectroscopy to demonstrate that TMPyP quenches the excited state of the large QDs on the microsecond time scale (Figure 3B and Figure 5). The exciton quenching on the nanosecond-to-microsecond time scales is due to a dynamic mechanism, whereby collisionally gated CT occurs between the QDs and freely diffusing TMPyP molecules. This observation provides further evidence for the involvement of both static and dynamic mechanisms in describing the overall quenching process, in agreement with other reported QD/molecular acceptor systems. ${ }^{8,36}$ The results of the nanosecond TA studies were consistent with the results of the PL quenching measurements, as shown in Figure 5 and Figure 2, respectively. The nanosecond TA studies revealed that the addition of $0.08 \mathrm{mM}$ TMPyP 


\section{WILEY-VCH}

resulted in the evolution of a new fast decay component and maintenance of approximately $67 \%$ of the QD population with an elongated lifetime of $0.98 \mu$ s. Similarly, the PL quenching measurements revealed that, upon the addition of $0.08 \mathrm{mM}$ TMPyP to PbS-1300 (green traces), approximately 64\% of the QD population maintained an active PL.

\subsection{The importance of Interfacial Electrostatic Interaction}

We argue that, although the $\mathrm{CT}$ from $\mathrm{PbS}$ QDs to TMPyP is facilitated by their interfacial band energy level alignment, this CT is based primarily on the prior availability of intimate contact through the electrostatic interaction between the two moieties as an additional driving force. To confirm this hypothesis, we evaluated the interaction between $\mathrm{PbS}$ QDs and a neutrally charged free-base porphyrin, 5,10,15,20-tetra(4-pyridyl)porphyrin (TPyP), under the same experimental conditions. In this case, whereas TPyP has energy levels similar to those of $\mathrm{TMPyP}^{35,37}$ it lacks the positive charges that provide electrostatic interaction with the TGcapped QDs. Figure 6 shows that the addition of TPyP to PbS-1300 did not induce any PL quenching, nor significantly change the fs-TA kinetics. The complete absence of PL quenching between large PbS QDs and TPyP, and the lack of any differences in the QD's lifetime upon TPyP addition (Figure S7), reveal the importance of electrostatic interaction as a key parameter in controlling the CT at QD interfaces and in providing a broader effective QD size range.

Moreover, the importance of the electrostatic interaction would be manifested if it can also facilitate $\mathrm{CT}$ at interfaces with a dis-favorable energy level alignment. Accordingly, we perform another control experiment for measuring the PL quenching upon the addition of an equally charged molecule that provides a dis-favorable energy level alignment with $\mathrm{PbS}$ QDs. For that purpose, we use ZnTMPyP, since the complexation of $\mathrm{Zn}^{+2}$ with porphyrin had been reported to induce a rise in the porphyrin LUMO level by $\sim 1.5 \mathrm{eV} ;^{38}$ which should greatly inhibit CT from the QDs to the porphyrin species. Interestingly, complete PL quenching was 


\section{WILEY-VCH}

recorded with three QD sizes (PbS-800, PbS-1300, and PbS-1500) upon the addition of ZnTMPyP (Figure S9), which suggests that the proximity of a cationic species could enhance charge transfer even with a slight dis-favorable-energy level alignment.

\section{Conclusion}

In summary, we report, for the first time, the possibility of extending the effective size range for $\mathrm{CT}$ at the $\mathrm{PbS}$ QDs interface, thus allowing the coupling of the faster electron injection rate of small QDs and the greater absorption range of large QDs to harvest different portions of the broad solar spectrum, overcoming one of the greatest challenges impeding the development of PbS QD-based solar cells. More specifically, we demonstrate that, by utilizing both the quantum confinement and the interfacial electrostatic interaction, one can now extend the effective QD size for CT to include most of the near-infrared spectral region, reaching beyond the previously reported cut-off CT bandgaps for PbS QDs. We believe that these findings shed light on the key variable components for designing QD-sensitized solar cells as well as other state-of-the-art devices that rely on interfacial CT processes.

\section{Experimental Section}

Quantum Dot Synthesis: The QD synthesis was performed in a three-neck round bottom flask with Schlenk line setting using lead oleate and TMS as precursors in ODE, according to Hines and Scholes. ${ }^{39}$ The TMS injection temperature was varied between $120-67{ }^{\circ} \mathrm{C}$ depending on the required QD size. After TMS injection, slow cooling was allowed to facilitate size focusing and help narrowing the size distribution. Once $60{ }^{\circ} \mathrm{C}$ was reached, $\mathrm{CdCl}_{2}$ treatment $\left(\mathrm{CdCl}_{2}\right.$-TDPA-Oleylamine) was introduced according to Ip et al.. ${ }^{5}$ Slow cooling was continued until $35^{\circ} \mathrm{C}$ is reached whereby acetone was added to precipitate the QDs from solution. After centrifugation, the OA-capped $\mathrm{CdCl}_{2}$-treated $\mathrm{PbS}$ QDs were purified twice by 


\section{WILEY-VCH}

dispersion in toluene and re-precipitation with acetone/methanol (1:1 volume ratio), and finally dissolved in octane $(150 \mathrm{mg} / \mathrm{mL})$.

Solution-Phase Ligand Exchange: The solution-phase ligand exchange was done according to a modified literature method. ${ }^{22}$ Shortly, $100 \mu \mathrm{L}$ of the OA-capped $\mathrm{CdCl}_{2}$-treated QDs $(150$ $\mathrm{mg} / \mathrm{mL})$ were dispersed in $4 \mathrm{~mL}$ of octane. The mixture was added to a solution of TG $(90 \mu \mathrm{L}$, $0.112 \mathrm{~g})$ in DMSO (4 mL). The biphasic system was vortexed vigorously for 30 seconds, resulting in the migration of QDs from non-polar to polar phase. After centrifugation at 5000 rpm for 1 minute, the polar phase was separated from the clear non-polar phase. The polar QD dispersion was rinsed four more times with an equal volume of octane, followed by vortexing, and centrifugation. Finally, acetonitrile was added in 4:1 volume ratio to induce precipitation and remove excess ligands, followed by centrifugation, and isolation. The TG-capped $\mathrm{CdCl}_{2}-$ treated QDs were then dried under vacuum overnight, dispersed in DMSO, and centrifuged at $15000 \mathrm{rpm}$ for five minutes to remove any aggregates.

Transmission Electron Microscopy (TEM): This was carried out on a TitanG2 80-300 instrument, FEI Co., Super Twin, x-FEG, operating at $300 \mathrm{kV}$. The PbS QDs dispersions in DMSO were deposited onto 300 mesh gold grids with holey carbon film, and dried in air for at least 4 hour before imaging.

Dynamic Light Scattering (DLS) and Zeta-Potential: The measurements were performed using a Zetasizer Nano (Malvern) at $20^{\circ} \mathrm{C}$ in a $1-\mathrm{cm}$ path length quartz cuvette, with backscattering and front-scattering detection modes, respectively. The size distributions are based on the hydrodynamic diameter of the QD dispersions in DMSO. 


\section{WILEY-VCH}

Photoelectron Spectroscopy: X-ray photoelectron spectroscopy (XPS) measurements were carried out in a ultrahigh vacuum chamber (UHV) Omicron chamber equipped with a SPHERA U7 hemispherical energy analyzer, using X-ray photons with an incident kinetic energy of $1486.6 \mathrm{eV}$ from a monochromated $\mathrm{Al} \mathrm{K} \alpha \mathrm{X}$-ray source with a total energy resolution of $0.1 \mathrm{eV}$. The survey spectrum was acquired at a pass energy of $40 \mathrm{eV}$, while the high resolution core level peaks for the various elements were obtained at a pass energy of 20 $\mathrm{eV}$. The photoelectrons were collected by the SPHERA U7 hemispherical energy analyzer with a 7 channel MCD detector, in Constant Analyzer Energy (CAE) mode. The spectra were referenced to the $\mathrm{C} 1 \mathrm{~s}$ core level peak with its $\mathrm{C}-\mathrm{C}$ chemical bond component neutralized at 285.0 eV. The QD dispersions in DMSO were deposited on tin-doped indium oxide (ITO) coated glass by drop-casting, followed by mild heating at $35^{\circ} \mathrm{C}$ to accelerate the drying process. As for ultraviolet photoelectron spectroscopy (UPS) measurements, the base pressure was maintained below $5 \times 10^{-9} \mathrm{mb}$. He I photons $(21.2 \mathrm{eV})$ were used to acquire the spectra at normal emission. The photon line width was $\sim 250 \mathrm{eV}$ and the minimum spot size was $\sim 1 \mathrm{~mm}$.

Steady-State Quenching Measurements: The stock TG-capped $\mathrm{CdCl}_{2}$-treated $\mathrm{PbS} \mathrm{QDs}$ were diluted with extra DMSO to maintain a constant concentration $\left(\sim 1.5 \times 10^{-6} \mathrm{M}\right)$, as determined by the optical density (OD) at the first exciton absorption peak (i.e. $1 \mathrm{~S}_{\mathrm{h}} \rightarrow 1 \mathrm{~S}_{\mathrm{e}}$ transition)

within a 1-cm path length quartz cuvette, where $\varepsilon_{\mathrm{pbs} \mathrm{QD}}=19600 * \mathrm{r}^{2.32} \cdot{ }^{40}$ Alternatively, the stock solutions were diluted with different concentrations of the porphyrin (TPyP or TMPyP in DMSO) while maintaining a fixed QDs concentration. The steady-state absorption spectra of these solutions were measured using a Cary 5000 UV-VIS-NIR spectrophotometer (Varian Inc.), while the steady-state photoluminescence spectra were measured using a Jobin-YvonHoriba Nanolog spectrofluorometer. 


\section{WILEY-VCH}

Transient Absorption (TA) Spectroscopy: The stock PbS QDs were diluted with extra DMSO,

or alternatively porphyrin (TPyP or TMPyP) in DMSO, while maintaining a fixed QD

concentration $\left(\sim 1.5 \times 10^{-6} \mathrm{M}\right)$ as determined by the OD at the first exciton absorption peak

within a 2-mm path length cuvette, where $\varepsilon_{\mathrm{pbs} \mathrm{QD}}=19600 * \mathrm{r}^{2.32} \cdot{ }^{40}$ All TA experiments were

conducted at room temperature, with constant sample stirring to avoid the photocharging of

QDs. ${ }^{41,42}$ The absorption spectrum of each sample measured before and after TA experiments

did not show any degradation. Further TA setup details are available in the SI.

\section{Supporting Information}

Supporting Information is available from the Wiley Online Library or from the author.

\section{Acknowledgements}

The work reported here was supported by King Abdullah University of Science and Technology (KAUST). A. El-Ballouli gratefully acknowledges Dr. Ahmed L. Abdelhady for his assistance with TEM measurements.

Received: ((will be filled in by the editorial staff))

Revised: ((will be filled in by the editorial staff)) Published online: ((will be filled in by the editorial staff))

(1) Hagfeldt, A.; Graetzel, M. Chem. Rev. 1995, 95, 49.

(2) Sambur, J. B.; Novet, T.; Parkinson, B. A. Science 2010, 330, 63.

(3) Tisdale, W. A.; Williams, K. J.; Timp, B. A.; Norris, D. J.; Aydil, E. S.; Zhu, X. Y. Science 2010, 328, 1543.

(4) El-Ballouli, A. O.; Alarousu, E.; Usman, A.; Pan, J.; Bakr, O. M.; Mohammed, O. F. ACS Photonics 2014, 1, 285.

(5) Ip, A. H.; Thon, S. M.; Hoogland, S.; Voznyy, O.; Zhitomirsky, D.; Debnath, R.; Levina, L.; Rollny, L. R.; Carey, G. H.; Fischer, A.; Kemp, K. W.; Kramer, I. J.; Ning, Z.; Labelle, A. J.; Chou, K. W.; Amassian, A.; Sargent, E. H. Nat. Nanotechnol. 2012, 7, 577. 2014, 13, 796 .

(6) Chuang, C.-H. M.; Brown, P. R.; Bulovic, V.; Bawendi, M. G. Nat. Mater.

(7) Pan, J.; El-Ballouli, A. O.; Rollny, L.; Voznyy, O.; Burlakov, V. M.; Goriely, A.; Sargent, E. H.; Bakr, O. M. ACS Nano 2013, 7, 10158.

(8) Knowles, K. E.; Malicki, M.; Weiss, E. A. J. Am. Chem. Soc. 2012, 134, 12470.

(9) Knowles, K. E.; Malicki, M.; Parameswaran, R.; Cass, L. C.; Weiss, E. A. J. Am. Chem. Soc. 2013, 135, 7264.

(10) Yang, Y.; Rodriguez-Cordoba, W.; Lian, T. J. Am. Chem. Soc. 2011, 133, 9246. 


\section{WILEY-VCH}

(11) El-Ballouli, A. O.; Alarousu, E.; Bernardi, M.; Aly, S. M.; Lagrow, A. P.; Bakr, O. M.; Mohammed, O. F. J. Am. Chem. Soc. 2014, 136, 6952.

(12) Choi, J. J.; Wenger, W. N.; Hoffman, R. S.; Lim, Y.-F.; Luria, J.; Jasieniak, J.; Marohn, J. A.; Hanrath, T. Adv. Mater. 2011, 23, 3144.

(13) Wang, X.; Koleilat, G. I.; Tang, J.; Liu, H.; Kramer, I. J.; Debnath, R.; Brzozowski, L.; Barkhouse, D. A. R.; Levina, L.; Hoogland, S.; Sargent, E. H. Nat. Photonics 2011, 5, 480 .

(14) Kramer, I. J.; Levina, L.; Debnath, R.; Zhitomirsky, D.; Sargent, E. H. Nano Lett. 2011, 11, 3701.

(15) Hyun, B.-R.; Zhong, Y.-W.; Bartnik, A. C.; Sun, L.; Abruna, H. D.; Wise, F. W.; Goodreau, J. D.; Matthews, J. R.; Leslie, T. M.; Borrelli, N. F. ACS Nano 2008, 2, 2206.

(16) Leventis, H. C.; O'Mahony, F.; Akhtar, J.; Afzaal, M.; O'Brien, P.; Haque, S. A. J. Am. Chem. Soc. 2010, 132, 2743.

(17) Gocalinska, A.; Saba, M.; Quochi, F.; Marceddu, M.; Szendrei, K.; Gao, J.; Loi, M. A.; Yarema, M.; Seyrkammer, R.; Heiss, W.; Mura, A.; Bongiovanni, G. J. Phys. Chem. Lett. 2010, 1, 1149.

(18) Zhao, H.; Liang, H.; Gonfa, B. A.; Chaker, M.; Ozaki, T.; Tijssen, P.; Vidal, F.; Ma, D. Nanoscale 2014, 6, 215.

(19) Guchhait, A.; Rath, A. K.; Pal, A. J. Sol. Energy Mater. Sol. Cells 2011, 95,

651.

(20) Tao, L.; Xiong, Y.; Liu, H.; Shen, W. Nanoscale 2014, 6, 931.

(21) Li, Z.; Wang, C. M.; Persaud, L.; Mallouk, T. E. J. Phys. Chem. 1988, 92, 2592.

(22) Fischer, A.; Rollny, L.; Pan, J.; Carey, G. H.; Thon, S. M.; Hoogland, S.;

Voznyy, O.; Zhitomirsky, D.; Kim, J. Y.; Bakr, O. M.; Sargent, E. H. Adv. Mater. 2013, 25, 5742.

(23) Katsiev, K.; Ip, A. H.; Fischer, A.; Tanabe, I.; Zhang, X.; Kirmani, A. R.; Voznyy, O.; Rollny, L. R.; Chou, K. W.; Thon, S. M.; Carey, G. H.; Cui, X.; Amassian, A.; Dowben, P.; Sargent, E. H.; Bakr, O. M. Adv. Mater. 2014, 26, 937.

$4,3777 / 1$.

(24) Feng, W.; Qin, C.; Shen, Y.; Li, Y.; Luo, W.; An, H.; Feng, Y. Sci. Rep. 2014,

(25) Wang, D.; Zhao, H.; Wu, N.; El Khakani, M. A.; Ma, D. J. Phys. Chem. Lett. 2010, 1,1030 .

(26) Brown, P. R.; Kim, D.; Lunt, R. R.; Zhao, N.; Bawendi, M. G.; Grossman, J. C.; Bulovic, V. ACS Nano 2014, 8, 5863.

(27) Lakowicz, J. R. Principles of Fluorescence Spectroscopy 3rd ed.; Springer: New York, 2006.

(28) Idowu, M.; Lamprecht, E.; Nyokong, T. J. Photochem. Photobiol., A 2008, 198,

7.

(29) Klimov, V. I. J. Phys. Chem. B 2000, 104, 6112.

(30) Fitzmorris, B. C.; Pu, Y.-C.; Cooper, J. K.; Lin, Y.-F.; Hsu, Y.-J.; Li, Y.;

Zhang, J. Z. ACS Appl. Mater. Interfaces 2013, 5, 2893.

(31) Kumar, M.; Neta, P.; Sutter, T. P. G.; Hambright, P. J. Phys. Chem. 1992, 96,

9571.

(32) Neta, P. J. Phys. Chem. 1981, 85, 3678.

(33) Mohammed, O. F.; Adamczyk, K.; Banerji, N.; Dreyer, J.; Lang, B.; Nibbering, E. T. J.; Vauthey, E. Angew. Chem., Int. Ed. 2008, 47, 9044.

(34) Mohammed, O. F.; Vauthey, E. J. Phys. Chem. A 2008, 112, 5804.

(35) Zhu, K.; Hu, X.; Ge, Q.; Sun, Q. Anal. Chim. Acta 2014, 812, 199.

(36) Mandal, S.; Rahaman, M.; Sadhu, S.; Nayak, S. K.; Patra, A. J. Phys. Chem. C 2013, 117, 3069 .

(37) Ohmori, Y.; Itoh, E.; Miyairi, K. Thin Solid Films 2006, 499, 369. 


\section{WILEY-VCH}

(38) Liao, M.-S.; Scheiner, S. J. Comput. Chem. 2002, 23, 1391.

(39) Hines, M. A.; Scholes, G. D. Adv. Mater. 2003, 15, 1844.

(40) Cademartiri, L.; Montanari, E.; Calestani, G.; Migliori, A.; Guagliardi, A.; Ozin, G. A. J. Am. Chem. Soc. 2006, 128, 10337.

(41) Hardman, S. J. O.; Graham, D. M.; Stubbs, S. K.; Spencer, B. F.; Seddon, E. A.; Fung, H.-T.; Gardonio, S.; Sirotti, F.; Silly, M. G.; Akhtar, J.; O'Brien, P.; Binks, D. J.; Flavell, W. R. Phys. Chem. Chem. Phys. 2011, 13, 20275.

(42) Beard, M. C.; Knutsen, K. P.; Yu, P.; Luther, J. M.; Song, Q.; Metzger, W. K.; Ellingson, R. J.; Nozik, A. J. Nano Letters 2007, 7, 2506.

(43) Kim, D. I.; Islam, M. A.; Avila, L.; Herman, I. P. J. Phys. Chem. B 2003, 107, 6318.

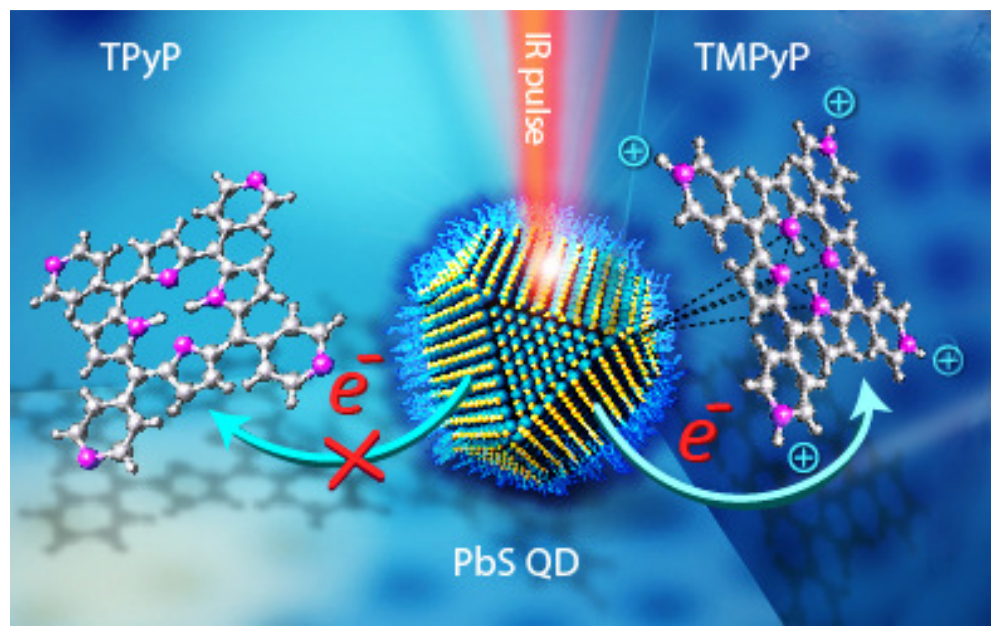

Scheme 1. The presence and absence of the CT process upon IR-pulse excitation from $\mathrm{PbS}$ QDs to TMPyP and TPyP, respectively. 

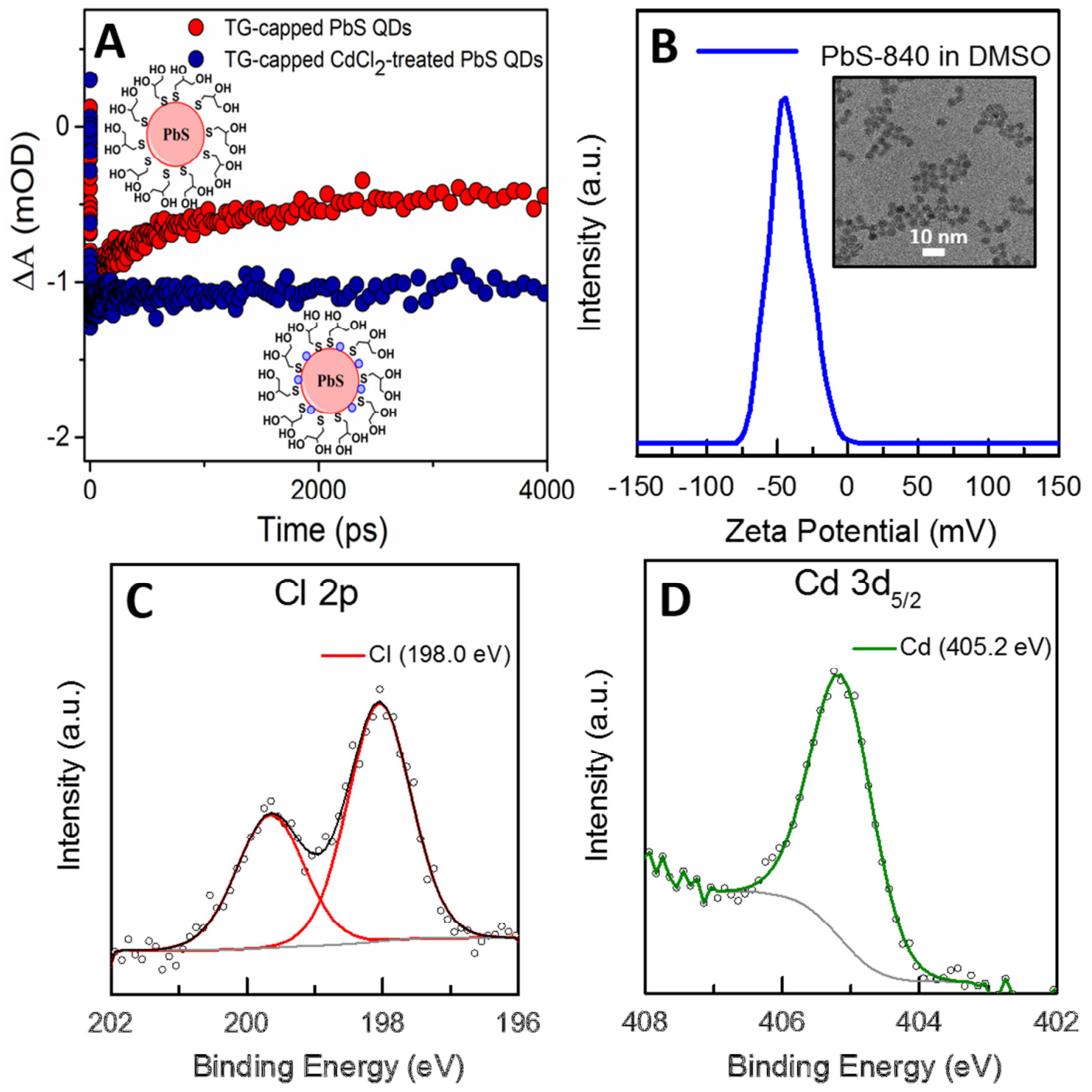

Figure 1. a) fs-TA kinetics traces at the GSB of similar-sized PbS QDs (PbS-1000) after ligand exchange to TG. Upon $\mathrm{CdCl}_{2}$-treatment, the QDs show acceptable recovery kinetics after ligand exchange. b) Zeta-potential measurement of TG-capped $\mathrm{CdCl}_{2}$-treated $\mathrm{PbS}$ QDs with a representative TEM image in the inset. c,d) XPS spectrum of PbS QD film showing $\mathrm{Cl}$ and Cd peaks enlarged, a survey XPS spectrum is available in the SI. 


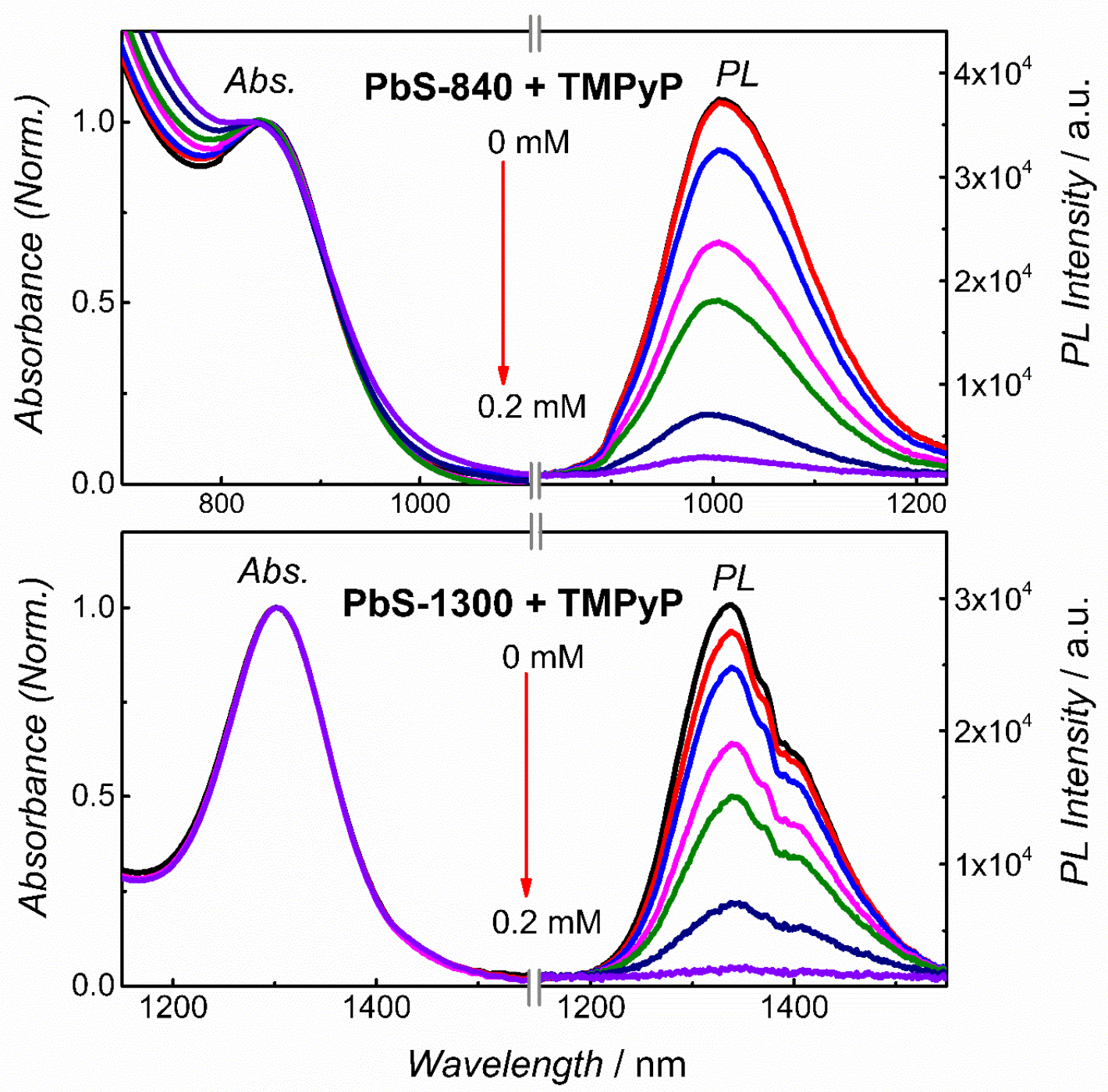

Figure 2. Steady-state absorption and PL ( $\lambda_{\mathrm{ex}}=700 \mathrm{~nm}$ and $\lambda_{\mathrm{ex}}=1000 \mathrm{~nm}$, respectively) spectra of PbS-840 and PbS-1300 upon the addition of different [TMPyP], in DMSO- $\mathrm{d}_{6}$. The green traces signify the addition of $0.08 \mathrm{mM}$ TMPyP. 

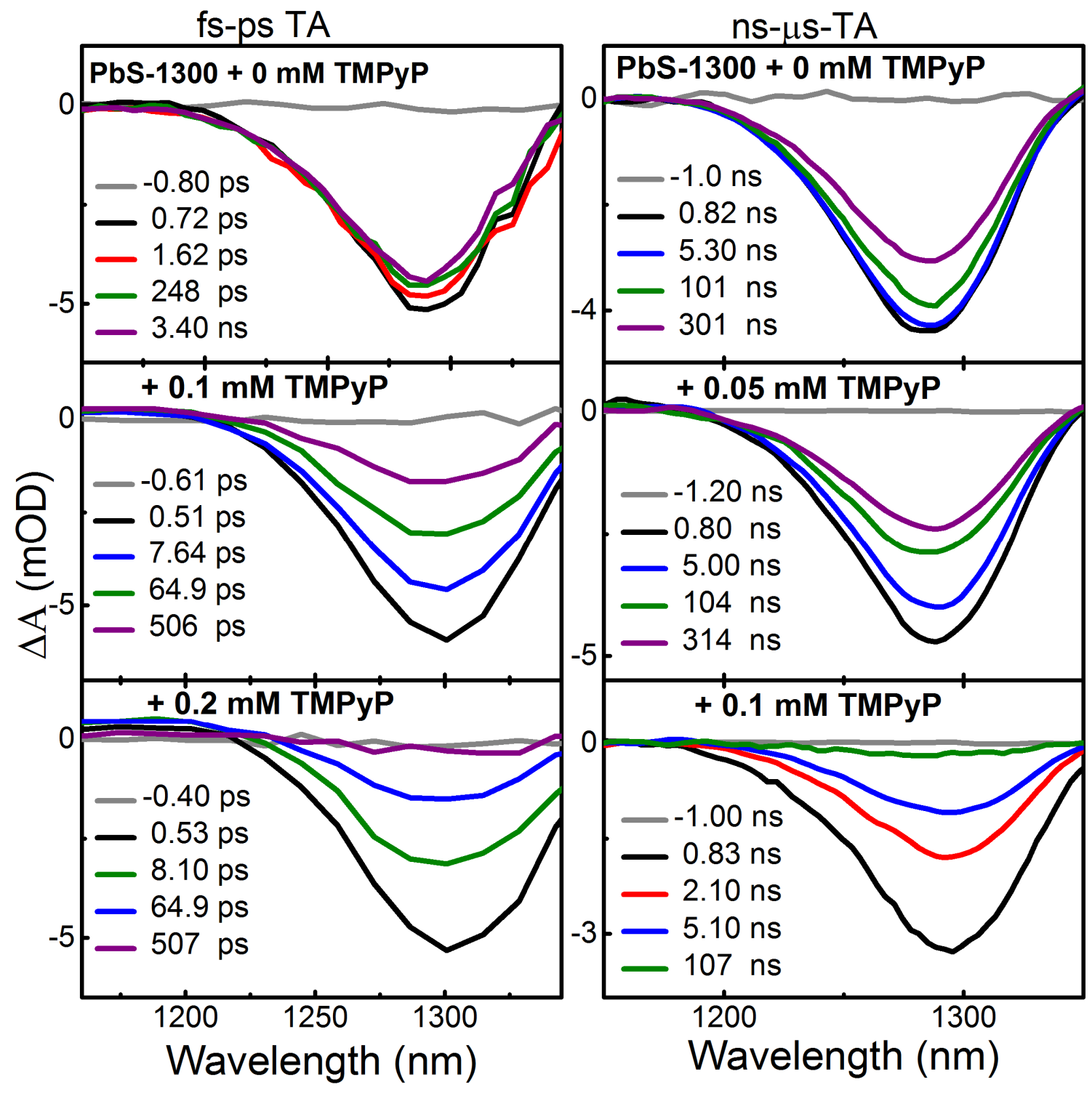

Figure 3. fs-ps TA (A) and ns- $\mu$ s TA (B) spectra of PbS-1300 upon the addition of different [TMPyP], with $\lambda_{\text {ex }}=1.3 \mathrm{E}_{\mathrm{g}}$ in DMSO- $\mathrm{d}_{6}$. 


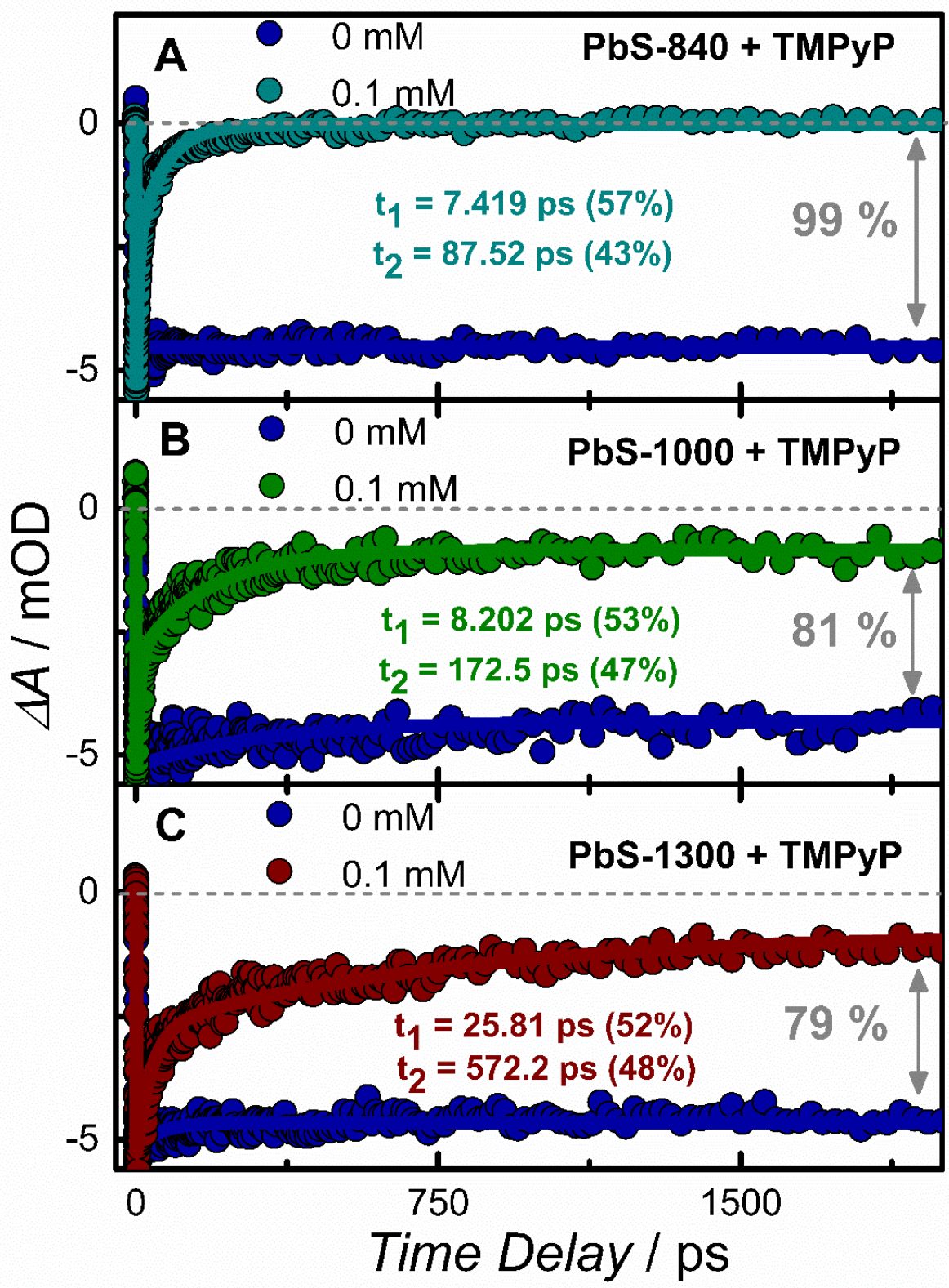

Figure 4. fs-TA kinetics traces at the GSB of PbS QDs with different sizes upon the addition of $0.1 \mathrm{mM}$ TMPyP, with $\lambda_{\mathrm{ex}}=1.3 \mathrm{E}_{\mathrm{g}}$ in DMSO- $\mathrm{d}_{6}$. 
WILEY-VCH

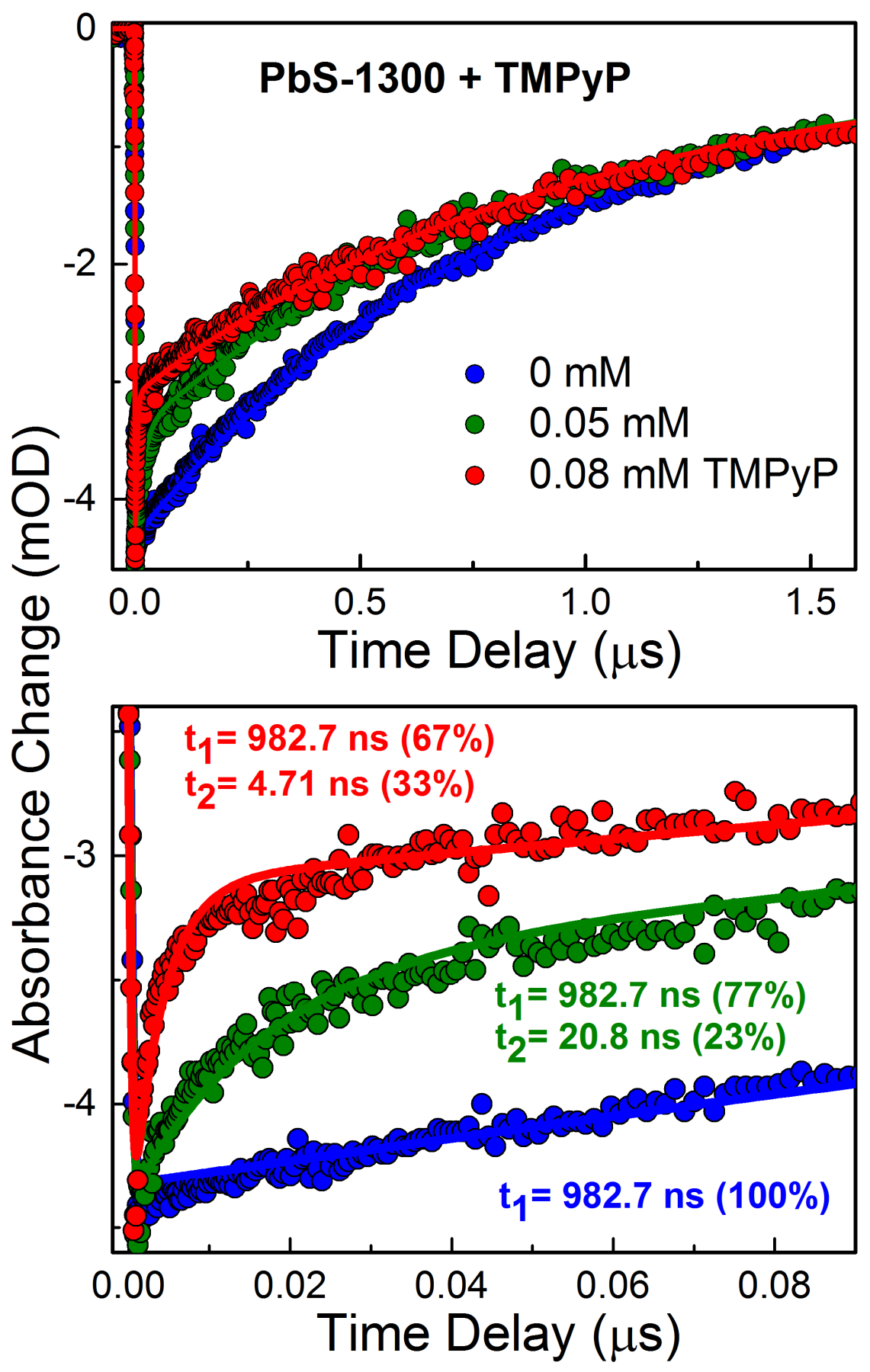

Figure 5. ns- $\mu$ s TA kinetics at the GSB for PbS-1300 with $\lambda_{\mathrm{ex}}=900 \mathrm{~nm}$, in the long timescale (Top). Upon TMPyP addition, a double exponential function arises as clarified in the short-time window (Bottom). By fixing $\mathrm{t}_{1}$ value to the lifetime of pure QDs (982.7 ns), it is clarified that its amplitude decreases upon TMPyP addition. 

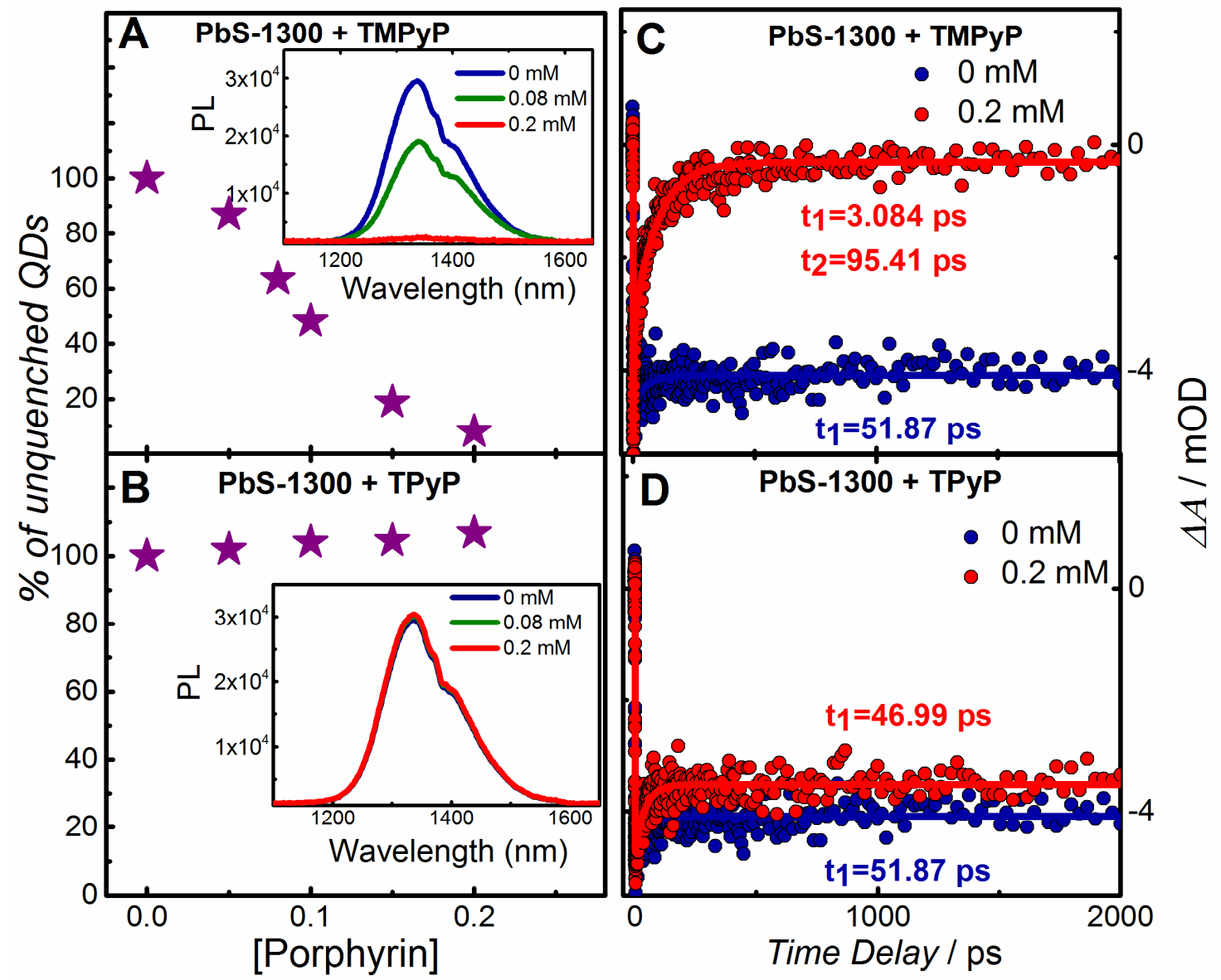

Figure 6. Plots of the percentage of unquenched QDs upon the addition of TMPyP (A) and TPyP (B), where the insets show the representative PL spectra. fs-TA kinetics at the GSB for PbS-1300 upon the addition of TMPyP (C) and TPyP (D). 
The interfacial electrostatic interaction between the positively-charged porphyrin and the negatively-charged QDs surface enables widening the effective bandgap $\left(E_{g}\right)$ range for charge transfer (CT) from PbS QDs. We show, for the first time, the occurance of an effective $\mathrm{CT}$ from large $\mathrm{PbS}$ QDs $\left(\mathrm{E}_{\mathrm{g}}<1 \mathrm{eV}\right.$ ) to positively-charged porphyrin, thus overcoming the previously-reported cut-off CT bandgaps at $\mathrm{PbS} \mathrm{QD}$ interfaces.

Ala'a O. El-Ballouli, Erkki Alarousu, Ahmad R. Kirmani, Aram Amassian, Osman M. Bakr, and Omar F. Mohammed*

Overcoming the Cut-off Charge Transfer Bandgaps at the PbS Quantum Dot Interface

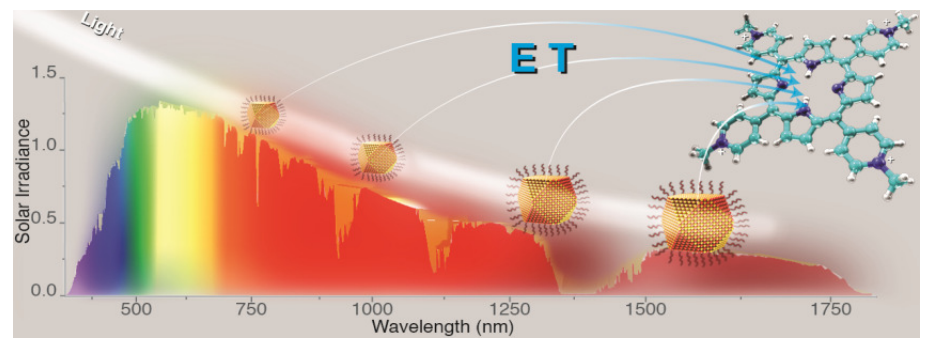


Copyright WILEY-VCH Verlag GmbH \& Co. KGaA, 69469 Weinheim, Germany, 2013.

\title{
Supporting Information
}

\author{
Overcoming the Cut-off Charge Transfer Bandgaps at the PbS Quantum Dot Interface \\ Ala'a O. El-Ballouli, Erkki Alarousu, Ahmad R. Kirmani, Aram Amassian, Osman M. Bakr, \\ and Omar F. Mohammed*
}

\section{$\underline{\text { Experimental Section }}$}

\section{Chemicals}

The precursors employed to prepare TG-capped $\mathrm{CdCl}_{2}$-treated PbS QDs were: lead(II) oxide powder (PbO, J. T. Baker, 99\%), oleic acid (OA, Alpha Aesar, technical grade 90\%), bis(trimethylsilyl) sulfide (TMS, Acros Organics, $95 \%$ purity), cadmium(II) chloride $\left(\mathrm{CdCl}_{2}\right.$, Alpha Aesar, anhydrous 99\%), tetradecylphosphonic acid (TDPA, Alfa Aesar, 98\%), oleylamine (Sigma-Aldrich, technical grade 70\%), and 1-thioglycerol (TG, Sigma-Aldrich, $\geq$ 97\%). The solvents used including: 1-octadecene (ODE, technical grade 90\%), toluene (anhydrous, 99.8\%), octane (anhydrous, $\geq 99 \%$ ), acetone (ACS reagent, $\geq 99.5 \%$ ), methanol (HPLC grade, > 99\%), acetonitrile (anhydrous, 99.8\%) and dimethylsulfoxide (DMSO, anhydrous, 99.9\%) were purchased from Sigma-Aldrich. Deuterated DMSO (DMSO-d $\mathrm{d}_{6}$, 99.5\% isotopic) was obtained from Alpha Aesar, while 5,10,15,20-tetra(4-pyridyl)porphyrin (TPyP) and 5,10,15,20-tetra(N-methyl-4-pyridyl)porphyrin tetrachloride (TMPyP) were purchased from Sigma-Aldrich and Frontier Scientific, respectively. ODE was degassed by pumping at $90{ }^{\circ} \mathrm{C}$ overnight before use, while the other chemicals were used as received without further purification. 


\section{WILEY-VCH}

\section{Transient Absorption (TA) Spectroscopy}

Femtosecond (fs) TA spectra were measured with a commercial broadband pump-probe setup (HELIOS, Ultrafast Systems) to probe in the visible (CMOS VIS spectrometer, 350-800 nm, $1.5 \mathrm{~nm}$ resolution at $9500 \mathrm{spectra} / \mathrm{s}$ ) and near-IR (InGaAs NIR spectrometer, $800-1600 \mathrm{~nm}$, $3.5 \mathrm{~nm}$ resolution at $7900 \mathrm{spectra} / \mathrm{s})$. The fundamental laser is provided by a Ti:Sapphire fs regenerative amplifier (Spectra-Physics Spitfire Pro 35F-XP) which produces 35 fs pulses at $800 \mathrm{~nm}$ with $4 \mathrm{~mJ}$ of energy/pulse, and a repetition rate of $1 \mathrm{kHz}$. A portion of the fundamental at $\lambda=800 \mathrm{~nm}(1 \mathrm{~mJ})$ is passed through frequency mixing stages and non-collinear difference-frequency generator (TOPAS-C stage) to tune its wavelength in the range $\lambda=240$ $2600 \mathrm{~nm}$; thus providing a selective pump beam for each sample. While the probe beam is provided by routing a small portion of the fundamental $(\sim 60 \mu \mathrm{J})$ via a delay line, adjustable pinholes, focusing lens, and variable neutral density filter to a crystal for white light continuum (WLC) generation; and further to the sample via a focusing mirror. A sapphire plate crystal and a proprietary crystal plate (Ultrafast Systems) are available to cover VIS and NIR ranges, respectively. The probe beam is collected by the spectrometer via collimating and focusing lenses, and wave pass filters to attenuate the white light around the Spitfire fundamental at $800 \mathrm{~nm}$. The computer-controlled delay line is used to vary the delay between the pump and probe pulses that allow TA measurements within a $5.8 \mathrm{~ns}$ time window. The time-resolved experiments were performed at low pump fluence to ensure that the photoexcited QDs are due to single-photon absorption. This was done by maintaining the average number of photons $\left(\mathrm{N}_{\mathrm{ph}}\right)$ absorbed per QD per pump pulse at the entrance face of our TA setup less than 0.2 . Pump and probe beams were adjusted to overlap spatially and temporally on the sample. All TA experiments were carried out at room temperature.

Nanosecond TA spectra were collected with a commercial spectrometer (EOS, Ultrafast Systems) that offers spectral measurements in an extended timescale (up to $400 \mu \mathrm{s}$ ) with 100 


\section{WILEY-VCH}

ps time resolution. The pump beam is generated in a method similar to fs-TA measurements described above. While the super-continuum probe beam $(400-1700 \mathrm{~m})$ is generated by focusing a diode laser into a photonic crystal fiber. For both Helios and EOS systems, the probe pulse is passed through a beam-splitter before reaching the sample, in order to split the beam into two-channels (sample and reference). In this way, a portion is sent directly to the sample, while the other is directed to the detector to act as a reference. This technique allows dividing out any fluctuations in the probe beam intensity during the experiment.

\section{Supporting Data}
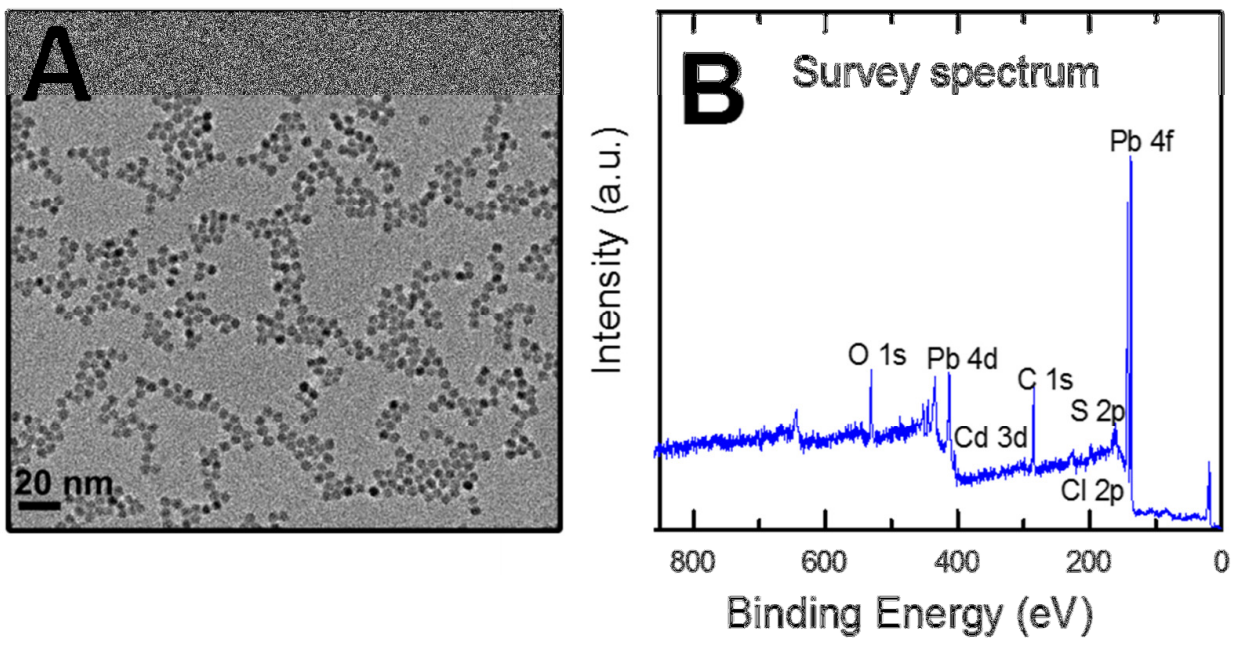

Figure S1. Representative TEM image of (TG-capped $\mathrm{CdCl}_{2}$-treated) PbS QDs (PbS-1300) showing the maintenance of a uniform shape and size-distribution after ligand exchange (A). Survey XPS spectrum of PbS QD film (B).

Table S1. Relative atomic ratios showing QDs composition as obtained by XPS.

\begin{tabular}{|l|lllllllll|}
\hline Elements & $\mathbf{P b}$ & $\mathbf{:}$ & $\mathbf{S}_{\text {core }}$ & $\mathbf{:}$ & $\mathbf{S}_{\mathbf{T G}}$ & $\mathbf{2}$ & $\mathbf{C l}$ & $\mathbf{1}$ & $\mathbf{C d}$ \\
\hline Atomic ratio & 1 & $:$ & 0.427 & $:$ & 0.165 & $:$ & 0.189 & $:$ & 0.037 \\
\hline
\end{tabular}



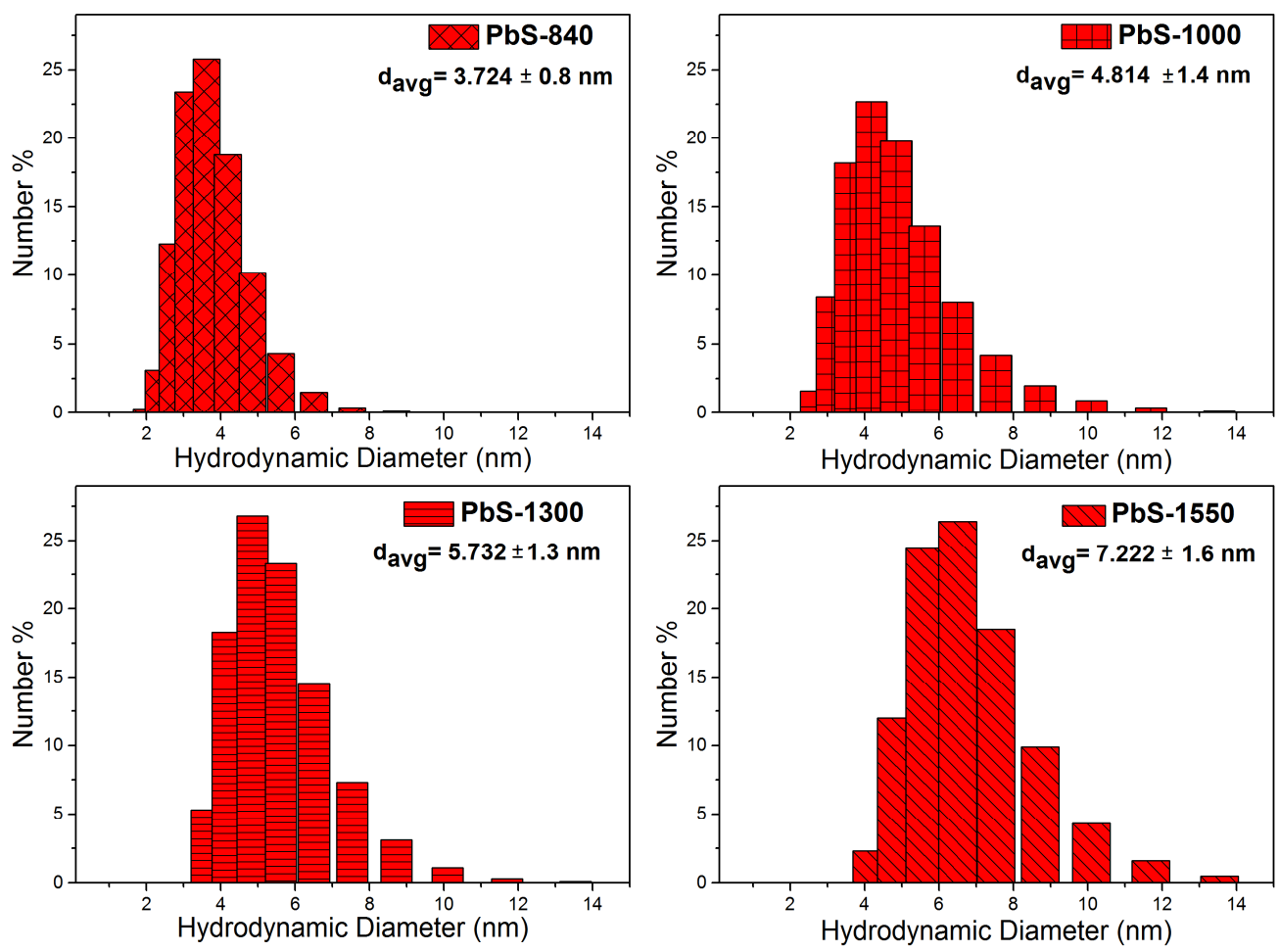

Figure S2. Size distributions of the PbS QDs used in this study based on the hydrodynamic diameter obtained by DLS.

Table S2. Comparison between the hydrodynamic diameter obtained by DLS, the calculated QD core diameter without the surface ligands, and the theoretical core diameter.

\begin{tabular}{|l|l|l|l|}
\hline QD Sample & $\begin{array}{l}\text { Obtained mean } \\
\text { hydrodynamic diameter } \\
\text { (HD) from DLS }\end{array}$ & $\begin{array}{l}\text { Calculated core diameter } \\
\text { (HD - 2 ligand layers of } \\
\text { TG, where TG = 0.76 nm) }\end{array}$ & $\begin{array}{l}\text { Theoretical core } \\
\text { diameter based on } \\
\text { Moreels } \text { et al. }{ }^{\text {2] }}\end{array}$ \\
\hline $\mathbf{P b S - 8 4 0}$ & $3.7 \pm 0.8 \mathrm{~nm}$ & $2.2 \pm 0.8 \mathrm{~nm}$ & $2.6 \mathrm{~nm}$ \\
\hline $\mathbf{P b S - 1 0 0 0}$ & $4.8 \pm 1.4 \mathrm{~nm}$ & $3.3 \pm 1.4 \mathrm{~nm}$ & $3.3 \mathrm{~nm}$ \\
\hline $\mathbf{P b S - 1 3 0 0}$ & $5.7 \pm 1.3 \mathrm{~nm}$ & $4.2 \pm 1.3 \mathrm{~nm}$ & $4.6 \mathrm{~nm}$ \\
\hline $\mathbf{P b S - 1 5 5 0}$ & $7.2 \pm 1.6 \mathrm{~nm}$ & $5.7 \pm 1.6 \mathrm{~nm}$ & $5.9 \mathrm{~nm}$ \\
\hline
\end{tabular}

The average core sizes of the QDs were further confirmed by analyzing the TEM images with Image $J$ software and measuring the diameter of $\sim 150$ particles. For instance, the average core diameters for PbS-1300 and PbS-1550 were found to be $4.8 \pm 0.5 \mathrm{~nm}$ and $6.1 \pm 0.4 \mathrm{~nm}$, respectively. 

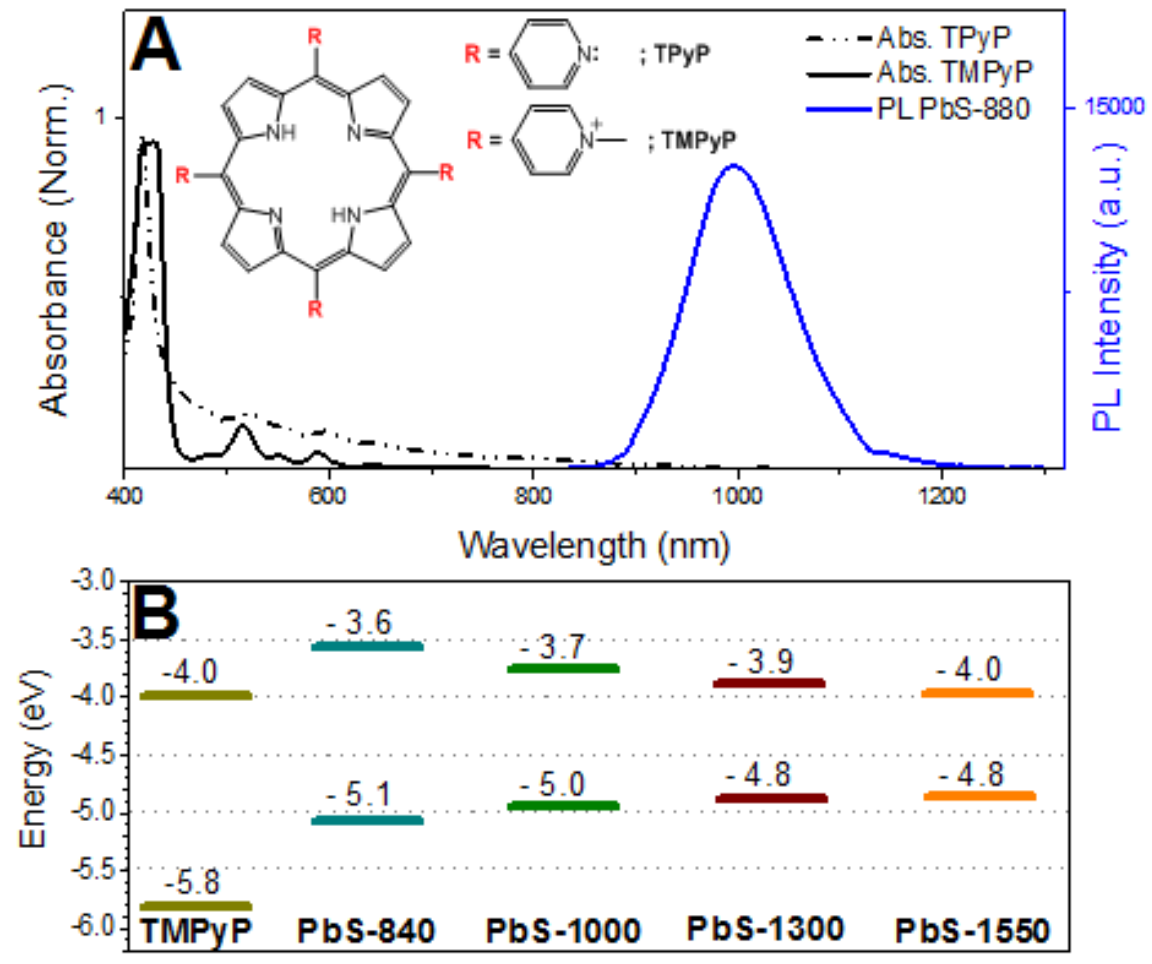

Figure S3. (A) Steady-state absorptions of TPyP and TMPyP (in DMSO- $\mathrm{d}_{6}$ ) show no spectral overlap with the PL spectra of small PbS QDs; which rules out the possibility of energy transfer. (B) A sketch of the energy band alignments of QD donors and TMPyP acceptor employed in this work. The valence band edges of the different QD sizes were determined by UPS. The conduction band edges were calculated by adding the optical bandgap energy, as determined from the first exciton absorption peak. The TMPyP HOMO and LUMO were obtained from literature. ${ }^{[3]}$ The binding energy values were rounded to the nearest $100 \mathrm{meV}$ value in accordance with the overall energy resolution.

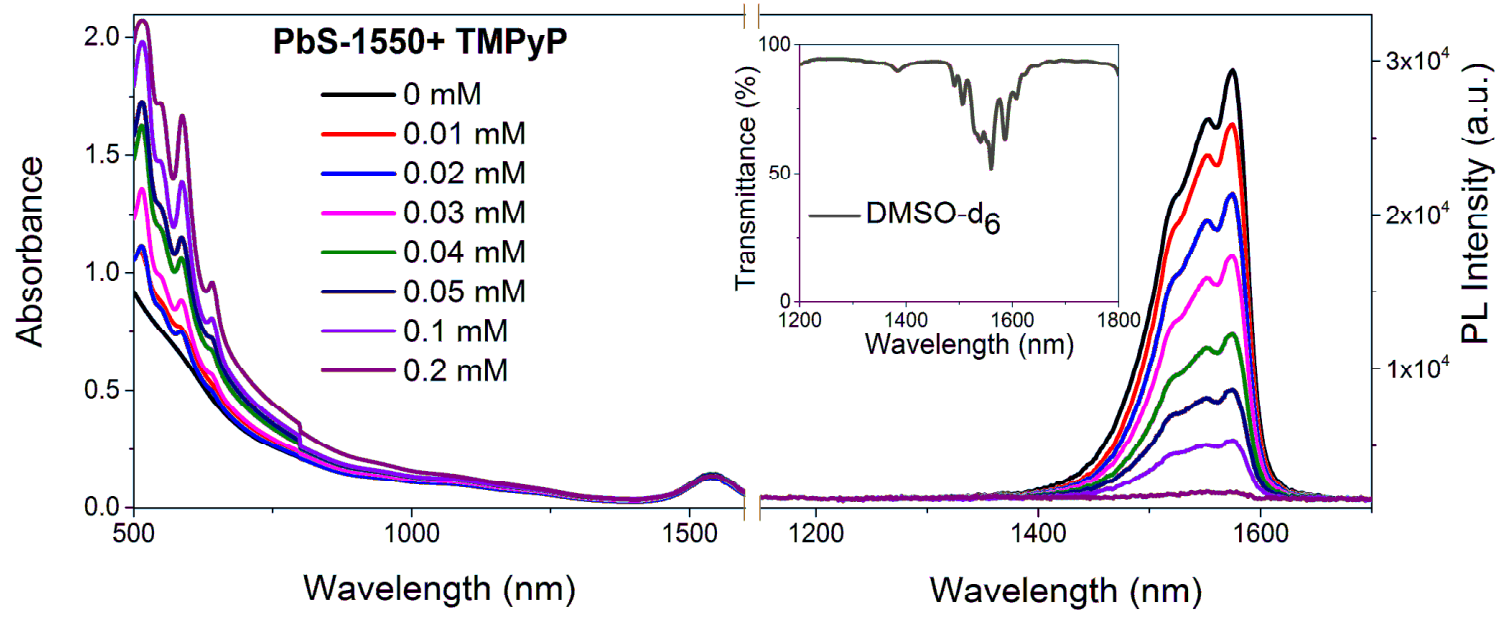

Figure S4. Steady-state absorption and PL spectra of PbS-1550 $\left(\sim 1 \times 10^{-6} \mathrm{M}\right)$ upon the addition of different [TMPyP], in DMSO- $\mathrm{d}_{6}$. The PL spectra were recorded upon excitation with $\lambda_{\mathrm{ex}}=1100 \mathrm{~nm}$. Note: the structures in PL spectra are a result of the solvent absorption in this region, as shown in the inset transmittance profile. 


\section{WILEY-VCH}

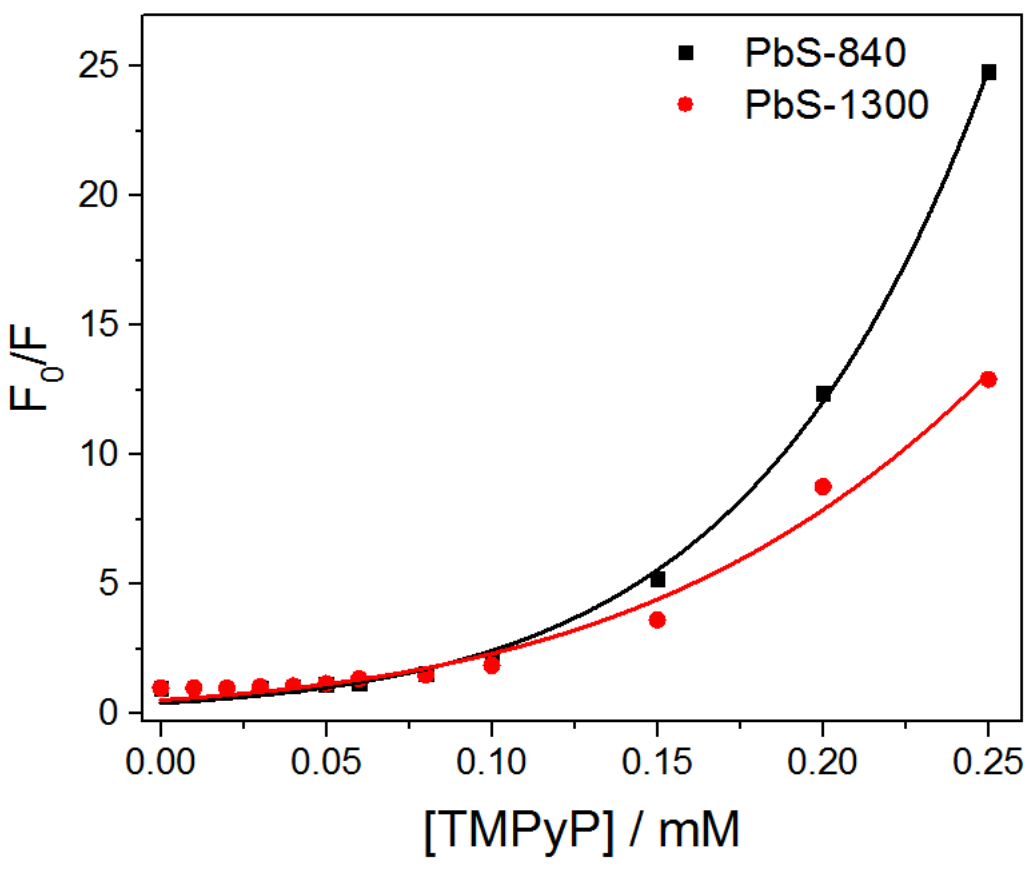

Figure S5. The Stern-Volmer (SV) plots for TMPyP addition to small and large QDs. Both plots reveal an upward curvature indicating the presence of complex quenching processes depending on the [TMPyP]. The plots could be based upon the equation: $\mathrm{F}_{0} / \mathrm{F}=\left(1+\mathrm{K}_{\mathrm{sv}}\right.$ [TMPyP] $)\left(1+\mathrm{K}_{\text {Assoc }}[\mathrm{TMPyP}]\right)$; thus presenting a combination of static and dynamic quenching. 


\section{WILEY-VCH}

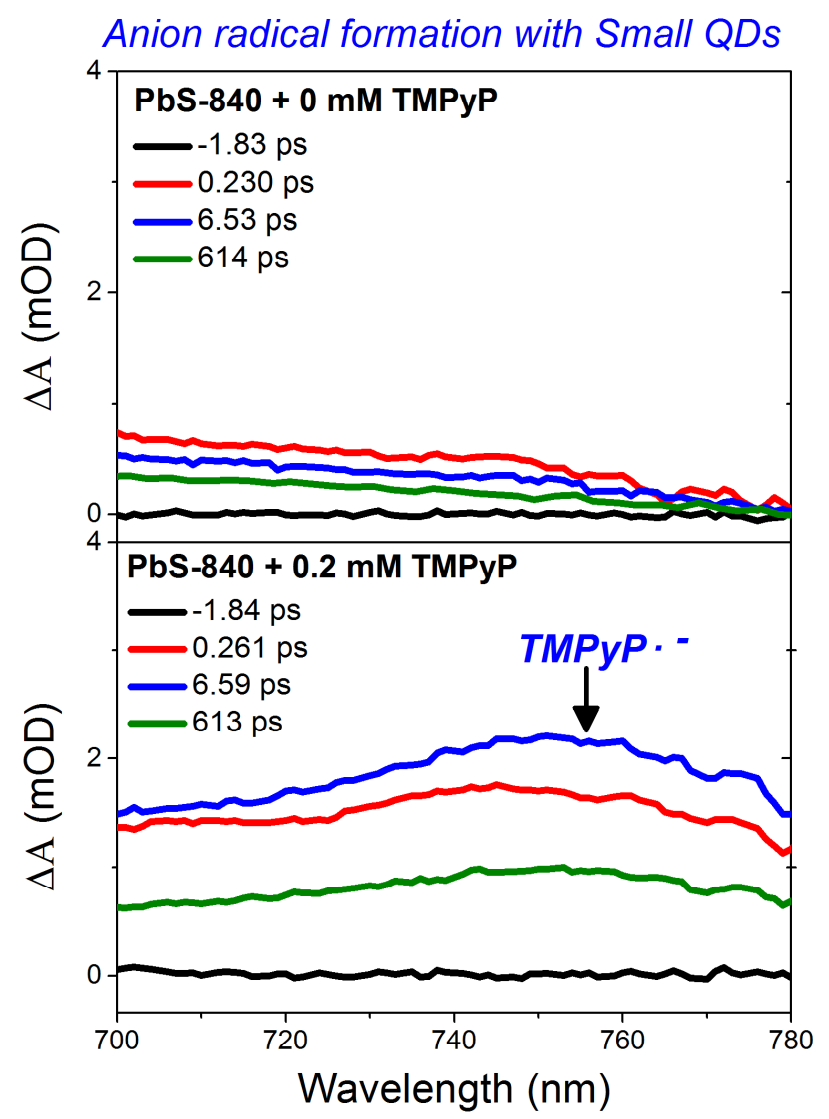

Anion radical formation with Large QDs

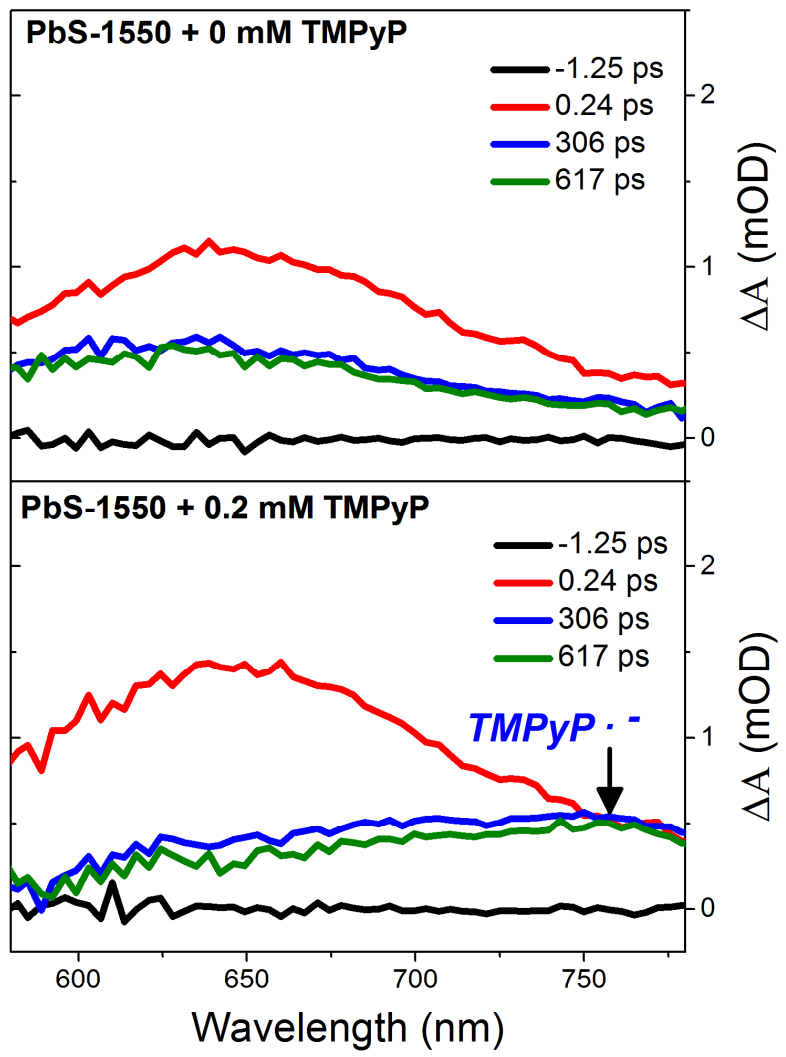

Figure S6. fs-TA spectra of $\mathrm{PbS}-840$ and $\mathrm{PbS}-1550$, in the visible region, with $\lambda_{\mathrm{ex}}=1.3 \mathrm{E}_{\mathrm{g}}$ in DMSO- $\mathrm{d}_{6}$. The spectra, in comparative timescales, show the evolution of TMPyP anion radical after excitation of small and large QDs in solution. 


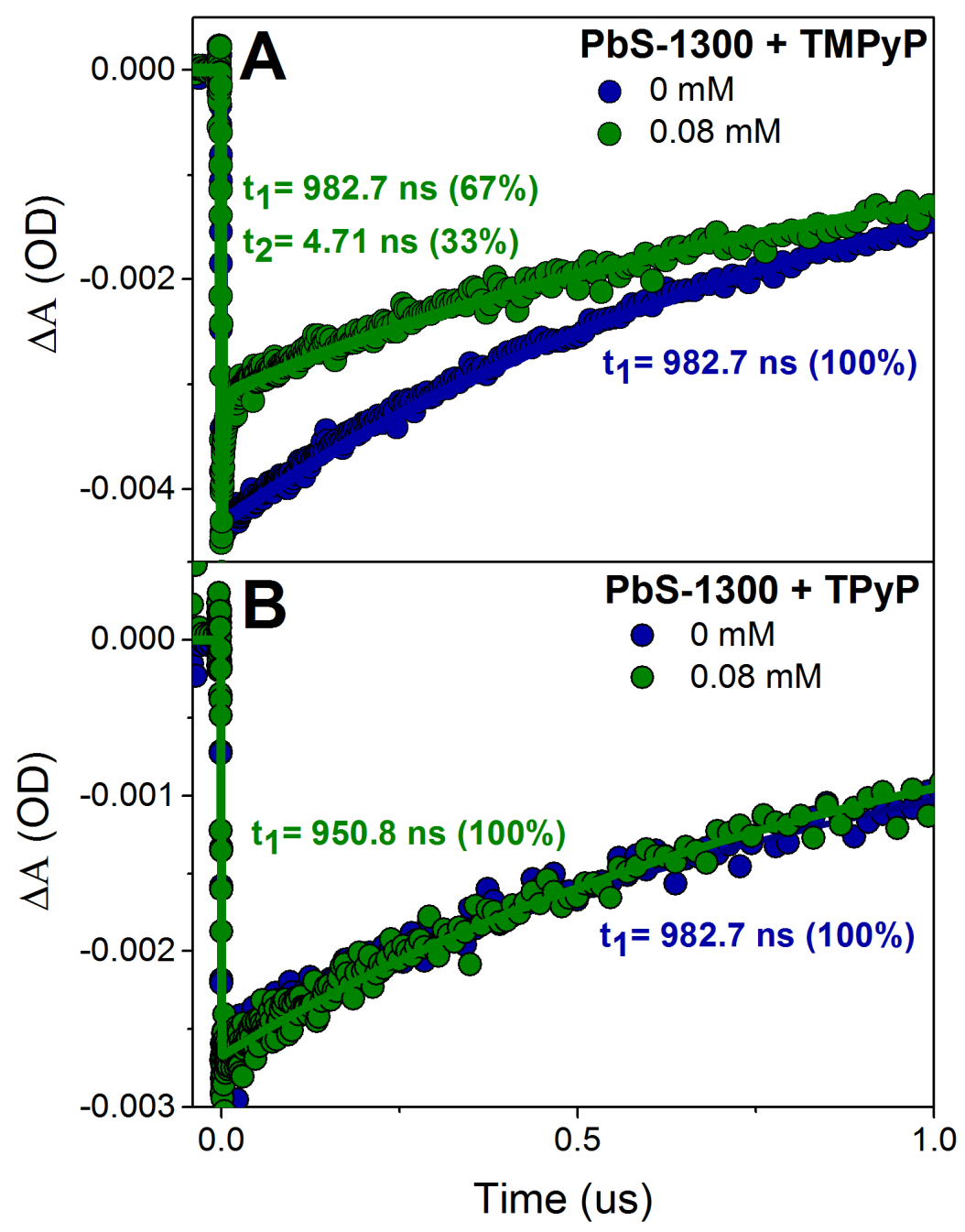

Figure S7. ns- $\mu$ s TA kinetics at the GSB for PbS-1300 with $\lambda_{\mathrm{ex}}=1.3 \mathrm{E}_{\mathrm{g}}$ upon the addition of TMPyP (A) and TPyP (B). The plot reveals the absence of the fast component evolution, and a nearly constant exciton lifetime when neutral TPyP is added. 


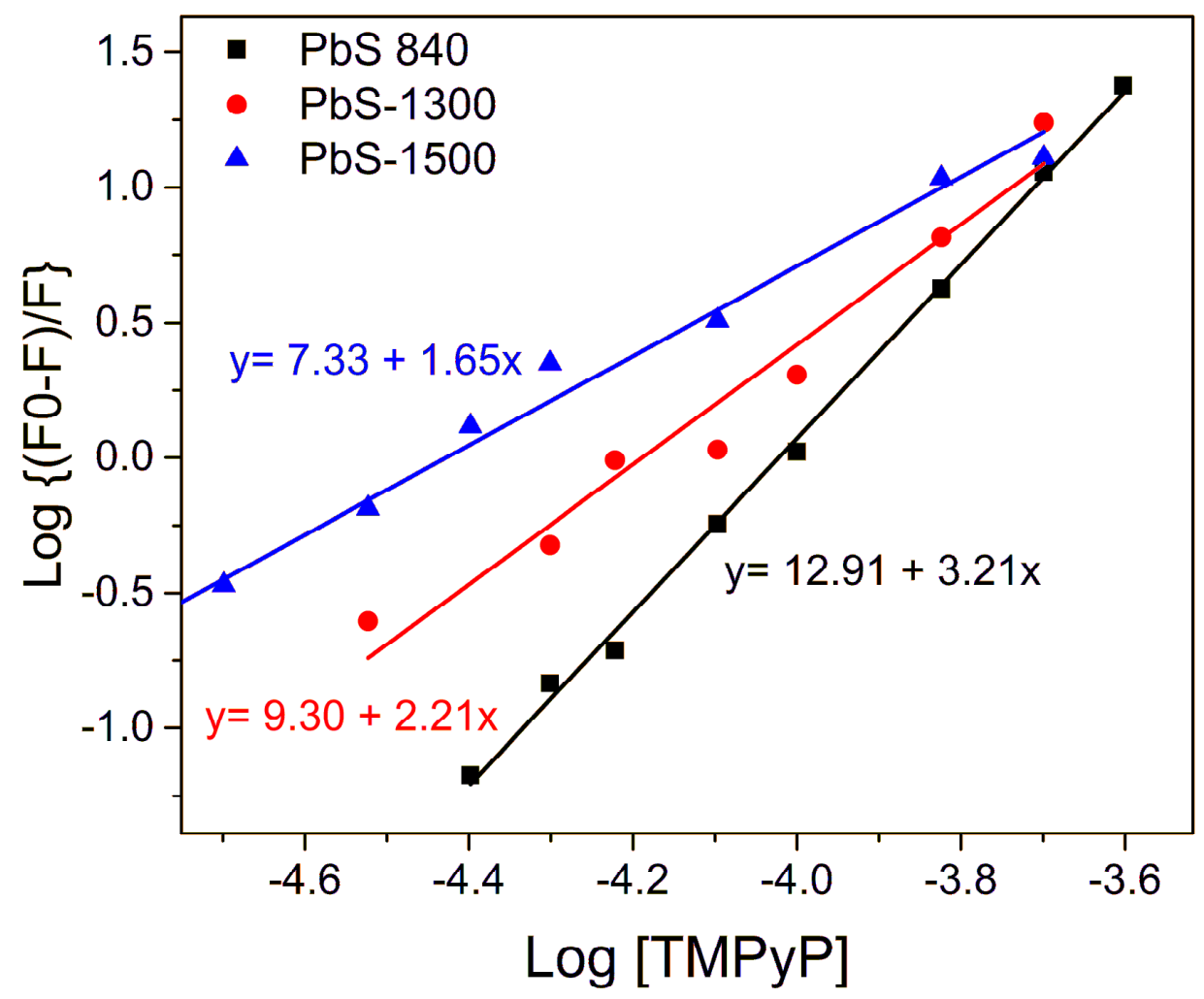

Figure S8. Modified Stern-Volmer plot representing the static quenching according to the equation: $\log \{(\mathrm{F} 0-\mathrm{F}) / \mathrm{F}\}=\log \mathrm{K}_{\mathrm{b}}+\mathrm{n} \log [\mathrm{Q}]$, for different QD sizes upon TMPyP addition. The plot reveals a significant decrease in the intercept as the QD size increases. This translates to a reduced binding constant; which could be the reason for the minimized blue shifts in the absorption and PL of large QDs with TMPyP.

Table S3. Calculated binding constants between different QD sizes and TMPyP based on the modified Stern-Volmer plots

\begin{tabular}{|l|l|l|l|}
\hline PbS size +TMPyP & Intercept $=\log \left(\mathbf{K}_{\mathbf{b}}\right)$ & Slope $=\mathbf{n}$ & $\mathbf{K}_{\mathbf{b}}\left(\mathbf{M}^{-\mathbf{1}}\right)$ \\
\hline $\mathrm{PbS}-840$ & 12.91 & 3.21 & $8.13 * 10^{12}$ \\
\hline $\mathrm{PbS}-1300$ & 9.30 & 2.21 & $2.00 * 10^{9}$ \\
\hline $\mathrm{PbS}-1500$ & 7.33 & 1.65 & $2.14 * 10^{7}$ \\
\hline
\end{tabular}



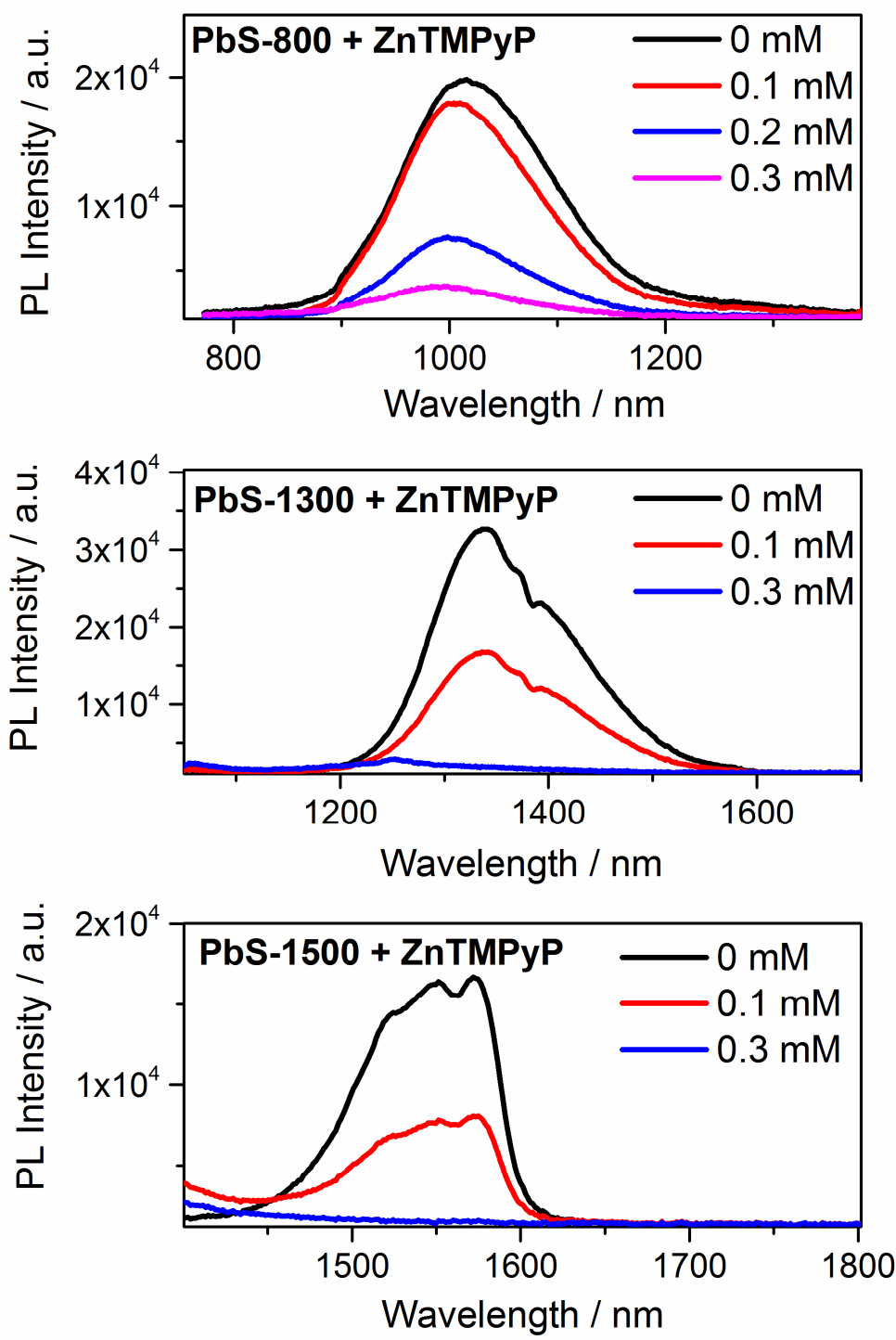

Figure S9. Steady-state absorption and PL spectra of different PbS QD sizes upon the addition of different [ZnTMPyP], in DMSO- $\mathrm{d}_{6}$. Complete PL quenching was recorded with the equally charged ZnTMPyP (compared to $\mathrm{H}_{2} \mathrm{TMPyP}$ ) although it provides a disfavorable energy level alignment with $\mathrm{PbS}$ QDs due the raise of the porphyrin LUMO upon the metal complexation. [4] 

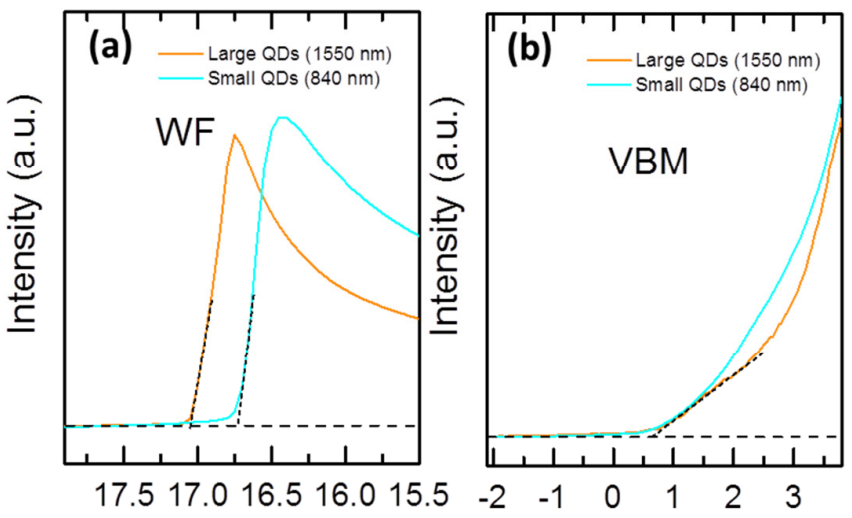

Binding Energy (eV)

Binding Energy (eV)

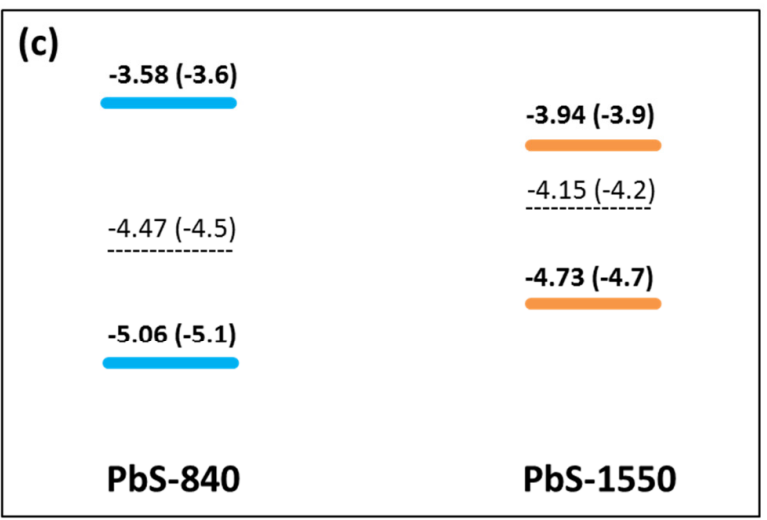

Figure S10: (a) Secondary electron cutoff and (b) Valence Band regions for the Large (1550 $\mathrm{nm})$ and Small $(840 \mathrm{~nm})$ are shown. Electronic band structures for the QDs are shown in (c). The conduction band minima are plotted by adding the optical band gaps. The values shown in brackets are the rounded figures for the energy levels, commensurate with the $\sim 0.1 \mathrm{eV}$ energy resolution of the analyser.

\section{$\underline{\text { Supporting References }}$}

[1] D. I. Kim, M. A. Islam, L. Avila, I. P. Herman, J. Phys. Chem. B 2003, 107, 6318-6323. [2] I. Moreels, K. Lambert, D. Smeets, D. De Muynck, T. Nollet, J. C. Martins, F. Vanhaecke, A. Vantomme, C. Delerue, G. Allan, Z. Hens, ACS Nano 2009, 3, 3023-3030.

[3] K. Zhu, X. Hu, Q. Ge, Q. Sun, Anal. Chim. Acta 2014, 812, 199-205.

[4] Liao, M. and Steve, S. J. Comput. Chem. 2002, 23, 1391-1403. 